\title{
ISOTROPIC POSITIVE DEFINITE FUNCTIONS ON SPHERES GENERATED FROM THOSE IN EUCLIDEAN SPACES
}

\author{
A Dissertation by \\ Zhihui Nie \\ Master of Science, Tianjin University of Technology and Education, 2014 \\ Bachelor of Science, Tianjin University of Technology and Education, 2011
}

Submitted to the Department of Mathematics, Statistics, and Physics and the faculty of the Graduate School of

Wichita State University

in partial fulfillment of

the requirements for the degree of

Doctor of Philosophy

May 2019 
(C) Copyright 2019 by Zhihui Nie

All Rights Reserved 


\section{ISOTROPIC POSITIVE DEFINITE FUNCTIONS ON SPHERES GENERATED FROM THOSE IN EUCLIDEAN SPACES}

The following faculty members have examined the final copy of this dissertation for form and content, and recommend that it be accepted in partial fulfillment of the requirement for the degree of Doctor of Philosophy with a major in Applied Mathematics.

Chunsheng Ma, Committee Chair

Tianshi Lu, Committee Member

Xiaomin $\mathrm{Hu}$, Committee Member

Adam Jaeger, Committee Member

Hyuck M. Kwon, Committee Member

Accepted for the College of Liberal Arts and Sciences

Andrew Hippisley, Dean

Accepted for the Graduate School

Kerry Wilks, Interim Dean 
DEDICATION

To my parents 


\section{ACKNOWLEDGEMENTS}

This dissertation would not have been possible without the guidance and help of several individuals who in one way or another contributed and extended their valuable assistance in the preparation and completion of this study. My first debt of gratitude must go to my advisor, Professor Chunsheng Ma, who was truly an inspiration. Without his invaluable guidance, this thesis would not have been possible. I would also like to to express my gratitude to Professor Daowei Ma, Professor Tianshi Lu, Professor Xiaomin Hu, Professor Adam Jaeger, Professor Hyuck M. Kwon and Professor Hua Liu for the contribution of their valuable time. In addition, I am grateful to acknowledge my friends in Wichita State University, J. Chen, X. Wei, L. Liang, Y. Wang, N. Nguyen, S. Rahmati, S. Zhang and F. Yan, for their friendship and warmth. Last but not the least, huge thank you to my parents, for their full supports. 


\section{ABSTRACT}

For a continuous function $g(x)$ on $[0, \pi]$ with $g(\pi)=0$, if it satisfies the inequality

$$
\int_{0}^{\pi} u^{\alpha+\frac{1}{2}} g(u) J_{\alpha-\frac{1}{2}}(x u) d u \geq 0, \quad x \geq 0
$$

it is shown in this thesis that

$$
\int_{0}^{\pi} g(u) P_{n}^{(\alpha)}(\cos u) \sin ^{2 \alpha} u d u \geq 0, \quad n \in \mathbb{N}_{0}
$$

where $\alpha$ is a nonnegative integer, and $J_{\nu}(x)$ and $P_{n}^{(\nu)}(x)$ denote the Bessel function and the ultraspherical polynomial, respectively. As a consequence, an isotropic and continuous positive definite function in the Euclidean space, if it is compactly supported, can be adopted as an isotropic positive definite function on a sphere. 


\section{TABLE OF CONTENTS}

Chapter $\quad$ Page

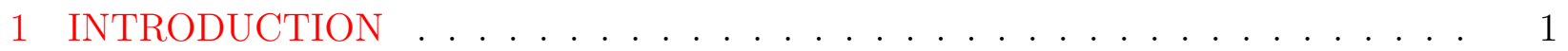

2 BACKGROUND INFORMATION . . . . . . . . . . . . . . . . 5

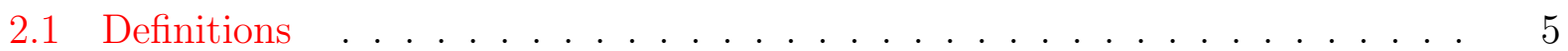

2.2 The Gamma Function . . . . . . . . . . . . . . . . . . . . 7

2.3 Orthogonal Polynomial . . . . . . . . . . . . . . . . . 8

2.4 Bessel Functions . . . . . . . . . . . . . . . . . . . . . . . . . . . . . . . 9

2.5 Abel-Summability . . . . . . . . . . . . . . . . . . . 10

3 ISOTROPIC POSITIVE DEFINITE FUNCTIONS IN EUCLIDEAN SPACE . . 12

3.1 Integral Transforms . . . . . . . . . . . . . . . . . . . . . . . . 12

3.2 Characterizations of Positive Definite Functions . . . . . . . . . . . . . 13

3.3 Schoenberg's Characterization . . . . . . . . . . . . . . . . . . 14

3.4 Properties of Positive Definite Functions . . . . . . . . . . . . . . 15

4 ISOTROPIC POSITIVE DEFINITE FUNCTIONS ON SPHERES . . . . . . . . 20

4.1 Some Classical Results Concerning Ultraspherical Polynomials . . . . . . . . 20

4.2 Schoenberg's Characterization . . . . . . . . . . . . . . . . 23

4.3 Recent Results . . . . . . . . . . . . . . . . . . . . . . . . . 24

5 ISOTROPIC POSITIVE DEFINITE FUNCTIONS ON SPHERES GENERATED FROM THOSE IN EUCLIDEAN SPACES . . . . . . . . . . . . . . 26

5.1 Main Results . . . . . . . . . . . . . . . . . . . . 26

5.2 Some Lemmas and Their Proofs . . . . . . . . . . . . . . . . . . . . . 30

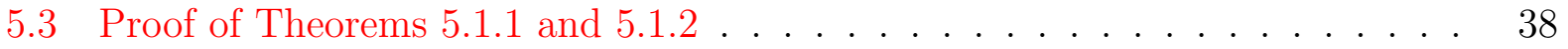

6 PLANS FOR FUTURE WORK . . . . . . . . . . . . . . . . . . . . . 41

REFERENCES . . . . . . . . . . . . . . . . . . . . . 42 


\section{CHAPTER 1 \\ INTRODUCTION}

There has run on renewed interest in isotropic positive definite functions in the Euclidean space $\mathbb{R}^{d}$ or on the sphere $\mathbb{S}^{d}$, with applications in approximation theory [4], [8], [10], [28], machine learning [20], probability, and spatial statistics [6], [15], [16], where $\mathbb{S}^{d}$ is the spherical

shell of radius 1 and center $\mathbf{0}$ in $\mathbb{R}^{d+1}$, i.e., $\mathbb{S}^{d}=\left\{\mathbf{x} \in \mathbb{R}^{d+1},\|\mathbf{x}\|=1\right\},\|\mathbf{x}\|$ is the Euclidean norm of $\mathbf{x}$, and $d$ is a positive integer.

An isotropic (radial, or spherically symmetric) function $g(\|\mathbf{x}\|): \mathbb{R}^{d} \rightarrow \mathbb{R}$, is said to be positive definite, if the inequality

$$
\sum_{i=1}^{n} \sum_{j=1}^{n} a_{i} a_{j} g\left(\left\|\mathbf{x}_{i}-\mathbf{x}_{j}\right\|\right) \geq 0
$$

holds for every $n \in \mathbb{N}$, any $a_{i} \in \mathbb{R}$ and $\mathbf{x}_{i} \in \mathbb{R}^{d}(i=1, \ldots, n)$, where $\mathbb{N}$ is the set of positive integers.

When $g(\|\mathbf{x}\|)$ is continuous in $\mathbb{R}^{d}$ or $g(\cdot)$ is continuous on $\mathbb{R}$, it is well-known [21] that $g(\|\mathbf{x}\|)$ is positive definite in $\mathbb{R}^{d}$ if and only if $g(x)$ admits a representation of the form

$$
g(x)=\int_{0}^{\infty}(x u)^{-\frac{d-2}{2}} J_{\frac{d-2}{2}}(x u) d F(u), \quad x \geq 0,
$$

where $F(x)$ is a bounded and nondecreasing function on $[0, \infty)$, and $J_{\nu}(x)$ denotes a Bessel function of order $\nu$.

As an example [2],

$$
g(x)=(a-x)_{+}^{\lambda}= \begin{cases}(a-x)^{\lambda}, & 0 \leq x \leq a, \\ 0, & x>a,\end{cases}
$$

makes $g(\|\mathbf{x}\|)$ an isotropic positive definite function in $\mathbb{R}^{d}$ whenever $\lambda \geq\left[\frac{d}{2}\right]$, where $a$ is a positive constant and $[x]$ denotes the greatest integer less than or equal to $x$.

For two points $\mathbf{x}$ and $\mathbf{y}$ on $\mathbb{S}^{d}$, their spherical (great circle, angular, or geodesic) distance, denotes by $\vartheta(\mathbf{x}, \mathbf{y})$, is the distance between $\mathbf{x}$ and $\mathbf{y}$ on the largest circle on $\mathbb{S}^{d}$ that passes 
through them. More precisely,

$$
\vartheta(\mathbf{x}, \mathbf{y})=\arccos \left(\mathbf{x}^{\prime} \mathbf{y}\right), \quad \mathbf{x}, \mathbf{y} \in \mathbb{S}^{d}
$$

where $\mathbf{x}^{\prime} \mathbf{y}$ denotes the inner product between $\mathbf{x}$ and $\mathbf{y}$. Since

$$
\|\mathbf{x}-\mathbf{y}\|^{2}=\|\mathbf{x}\|^{2}+\|\mathbf{y}\|^{2}-2 \mathbf{x}^{\prime} \mathbf{y}=2-2 \mathbf{x}^{\prime} \mathbf{y}
$$

another expression for $\vartheta(\mathbf{x}, \mathbf{y})$ in terms of the Euclidean distance $\|\mathbf{x}-\mathbf{y}\|$ is

$$
\vartheta(\mathbf{x}, \mathbf{y})=\arccos \left(1-\frac{1}{2}\|\mathbf{x}-\mathbf{y}\|^{2}\right), \quad \mathbf{x}, \mathbf{y} \in \mathbb{S}^{d}
$$

Evidently, $0 \leq \vartheta(\mathbf{x}, \mathbf{y}) \leq \pi$

A function $g\left(\vartheta\left(\mathbf{x}_{1}, \mathbf{x}_{2}\right)\right)$, or $g(\vartheta)$ for simplicity, is called an isotropic positive definite function on $\mathbb{S}^{d}$ if

$$
\sum_{i=1}^{n} \sum_{j=1}^{n} a_{i} a_{j} g\left(\vartheta\left(\mathbf{x}_{i}, \mathbf{x}_{j}\right)\right) \geq 0
$$

holds for every $n \in \mathbb{N}$, any $a_{i} \in \mathbb{R}$ and $\mathbf{x}_{i} \in \mathbb{S}^{d}, i=1, \ldots, n$.

Thus, an isotropic positive function on a sphere depends on its arguments via the spherical distance $\vartheta\left(\mathbf{x}_{1}, \mathbf{x}_{2}\right)$, or equivalently, via the inner product $\mathbf{x}_{1}^{\prime} \mathbf{x}_{2}$.

For a continuous function $g(\vartheta)$ on $[0, \pi]$, it is positive definite on $\mathbb{S}^{d}$ if and only if it can be expressed [22] as

$$
g(\vartheta)=\sum_{n=0}^{\infty} b_{n} P_{n}^{\left(\frac{d-1}{2}\right)}(\cos \vartheta), \quad \vartheta \in[0, \pi]
$$

where $\left\{b_{n}, n \in \mathbb{N}_{0}\right\}$ is a sequence of nonnegative constants, $\sum_{n=0}^{\infty} b_{n} P_{n}^{\left(\frac{d-1}{2}\right)}(1)$ converges, $P_{n}^{(\nu)}(x)$ denotes the Gagenbauer or ultraspherical polynomial [25] of degree $n$, and $\mathbb{N}_{0}$ is the set of nonnegative integers. In other words, $g(\vartheta)$ is positive definite on $\mathbb{S}^{d}$ if and only if

$$
b_{n}=\int_{0}^{\pi} g(\vartheta) P_{n}^{\left(\frac{d-1}{2}\right)}(\cos \vartheta) \sin ^{d-1} \vartheta d \vartheta \geq 0, \quad n \in \mathbb{N}_{0}
$$

and $\sum_{n=0}^{\infty} b_{n} P_{n}^{\left(\frac{d-1}{2}\right)}(1)$ converges. For $d \geq 2$, the convergence of $\sum_{n=0}^{\infty} b_{n} P_{n}^{\left(\frac{d-1}{2}\right)}(1)$ is equivalent to that of $\sum_{n=0}^{\infty} n^{d-2} b_{n}[6]$. 
A question of interest is: if a function $g(x)$ on $[0, \infty)$ makes $g(\|\mathbf{x}\|)$ an isotropic positive definite function in $\mathbb{R}^{d}$, would it also make $g(\vartheta)$ an isotropic positive definite function on $\mathbb{S}^{d}$ ? Such a question may be answered on a case-by-case basis. A counterexample is $g(x)=$ $\exp \left(-x^{2}\right), x \geq 0$, it is known that $g(\|\mathbf{x}\|)$ is positive definite in all dimensions, but $g(\vartheta)$ is not positive definite even on $\mathbb{S}^{1}$. On the other hand, a stimulating example is (1.0.2), for which it is shown in [4] that $g(\vartheta)$ is an isotropic positive definite function on $\mathbb{S}^{d}$ if $d \leq 7$, but, actually, the restriction $d \leq 7$ can be removed [28]. More interestingly, for $\alpha, \beta \in \mathbb{N}_{0}$, and $\max (\alpha, \beta)>0, \mathrm{Xu}[28]$ proves that

$$
\int_{0}^{a} g(\vartheta) P_{n}^{\left(\alpha-\frac{1}{2}, \beta-\frac{1}{2}\right)}(\cos \vartheta)\left(\sin \frac{\vartheta}{2}\right)^{2 \alpha}\left(\cos \frac{\vartheta}{2}\right)^{2 \beta} d \vartheta>0, \quad n \in \mathbb{N},
$$

for every $a \in(0, \pi]$ and $\lambda \geq \alpha+1$, where $P_{n}^{(\alpha, \beta)}(x)$ is the Jacobi polynomial [25] of degree $n$. When $\alpha=\beta, P_{n}^{(\alpha, \beta)}(x)$ reduces to the ultraspherical polynomial, with

$$
P_{n}^{(\alpha)}(x)=\frac{\Gamma\left(\alpha+\frac{1}{2}\right) \Gamma(n+2 \alpha)}{\Gamma(2 \alpha) \Gamma\left(n+\alpha+\frac{1}{2}\right)} P_{n}^{\left(\alpha-\frac{1}{2}, \alpha-\frac{1}{2}\right)}(x), \quad n \in \mathbb{N}_{0}, \alpha>-\frac{1}{2} ;
$$

see, e.g., (4.7.1) of [25].

A simple but important feature of the function (1.0.2) is that it is of compact support and takes nonnegative values. This motivates us to consider the following question: for a compactly supported function $g(x)$ on $[0, \infty)$, if it makes $g(\|\mathbf{x}\|)$ an isotropic positive definite function in $\mathbb{R}^{d}$, will it also make $g(\vartheta)$ an isotropic positive definite function on $\mathbb{S}^{d}$ ? The answer is yes, in a particualr case $d=1$ or 3 , but, as is conjectured in [15], it would be of interest to see whether such a result holds for a general $d$, for which a difficulty arises when one deals with the connection between the two bases, the Bessel functions for $\mathbb{R}^{d}$ and ultraspherical polynomials for $\mathbb{S}^{d}$. A useful bridge for the connection employed in [28] is Theorem 8.1 of [25], which is generalized in Theorem 5.1.3 below for us to answer the above question.

In this thesis we develop isotropic positive definite functions on odd dimensional spheres from those in Euclidean spaces. The rest of the thesis is organized as follows. We state the background information in chapter 2. The characterizations of isotropic positive definite functions on $\mathbb{R}^{d}$ were reviewed in chapter 3 . We discuss the characterizations of positive 
definite functions on $\mathbb{S}^{d}$ in chapter 4 . We introduce and prove our main result in chapter 5. There is an open question conjectured in chapter 6 for further research. 


\section{CHAPTER 2}

\section{BACKGROUND INFORMATION}

\subsection{Definitions}

In this expository section, we list the definitions of isotropic functions in $\mathbb{R}^{d}$ and on $\mathbb{S}^{d}$, and those of positive definite functions in $\mathbb{R}^{d}$ and on $\mathbb{S}^{d}$, as well as their connections to positive definite matrices.

Definition 2.1.1. (i) A function $G: \mathbb{R}^{d} \times \mathbb{R}^{d} \rightarrow \mathbb{R}$ is said to be isotropic, radial or spherical symmetric if there exists a function $g:[0, \infty) \rightarrow \mathbb{R}$ such that

$$
G(\mathbf{x}, \mathbf{y})=g(\|\mathbf{x}-\mathbf{y}\|)
$$

for all $\mathbf{x}, \mathbf{y} \in \mathbb{R}^{d}$.

(ii) A function $G: \mathbb{R}^{d} \rightarrow \mathbb{R}$ is said to be isotropic, radial or spherical symmetric if there exists a function $g:[0, \infty) \rightarrow \mathbb{R}$ such that

$$
G(\mathbf{x})=g(\|\mathbf{x}\|)
$$

for all $\mathbf{x} \in \mathbb{R}^{d}$.

Definition 2.1.2. A complex-valued function $g(\mathbf{x}, \mathbf{y})$ of two arbitrary points of $\mathbb{R}^{d}$ is called positive definite, if it enjoys the following properties:

(i) Hermitian symmetry

$$
g(\mathbf{x}-\mathbf{y})=\overline{g(\mathbf{x}-\mathbf{y})}
$$

(ii) For any finite set of $\mathbf{x}_{j} \in \mathbb{R}^{d}$ and complex numbers $z_{j}$ (with conjugate complex $\bar{z}_{j}$ ), $1 \leq j \leq n$, we have

$$
\sum_{j=1}^{n} \sum_{k=1}^{n} g\left(\mathbf{x}_{j}-\mathbf{x}_{k}\right) z_{j} \bar{z}_{k} \geq 0 .
$$

If the quadratic form (2.1.1) is zero only for $z \equiv 0$, then $g$ is called strictly positive definite. 
Here, we introduce a general definition for complex-valued positive definite functions. The reason for this is that it allows us to use techniques such as Fourier transforms more naturally in Chapter 3. However, we will see that for even, real-valued functions it suffices to investigate the quadratic form only for real numbers $z \in \mathbb{R}$.

Definition 2.1.3. A $d \times d$ symmetric real matrix $M$ is said to be positive definite if $\mathbf{x}^{\prime} M \mathbf{x} \geq 0$ for all $\mathbf{x} \in \mathbb{R}^{d}$.

Strictly positive definite matrices are defined similarly, except that the above scalar $\mathbf{x}^{\prime} M \mathbf{x}$ is positive for every $\mathbf{x} \neq 0$.

Now, we define positive definite functions by using positive definite matrices.

Definition 2.1.4. A function $G: \mathbb{R}^{d} \times \mathbb{R}^{d} \rightarrow \mathbb{R}$ is said to be positive definite if for any $N \in \mathbb{N}$ and any finite set of points $\left\{\mathbf{x}_{1}, \cdots, \mathbf{x}_{N}\right\}$ in $\mathbb{R}^{d}$, the $N \times N$ matrix

$$
G\left[X_{N}\right]=\left[G\left(\mathbf{x}_{i}, \mathbf{x}_{j}\right)\right]_{i, j=1}^{N}
$$

is positive definite. It is said to be strictly positive definite if the matrix $G\left[X_{N}\right]$ is strictly positive definite.

Definition 2.1.4 is equivalent to definition 2.1.5 (i).

Definition 2.1.5. (i) A function $G: \mathbb{R}^{d} \times \mathbb{R}^{d} \rightarrow \mathbb{R}$ is said to be positive definite, if the inequality

$$
\sum_{i=1}^{n} \sum_{j=1}^{n} a_{i} a_{j} G\left(\mathbf{x}_{i}, \mathbf{x}_{j}\right) \geq 0
$$

holds for every $n \in \mathbb{N}$, any $\mathbf{a}_{i} \in \mathbb{R}$, and any $\mathbf{x}_{i} \in \mathbb{R}^{d}(i=1, \cdots, n)$.

(ii) An isotropic function $g(x): \mathbb{R}^{d} \rightarrow \mathbb{R}$, is said to be positive definite, if the inequality

$$
\sum_{i=1}^{n} \sum_{j=1}^{n} a_{i} a_{j} g\left(\left\|\mathbf{x}_{i}-\mathbf{x}_{j}\right\|\right) \geq 0
$$

holds for every $n \in \mathbb{N}$, any $a_{i} \in \mathbb{R}$ and $\mathbf{x}_{i} \in \mathbb{R}^{d}(i=1, \ldots, n)$. 
Definition 2.1.6. A function $G: \mathbb{S}^{d} \times \mathbb{S}^{d} \rightarrow \mathbb{R}$ is said to be isotropic, if there exists a function $g:[0, \pi] \rightarrow \mathbb{R}$ such that $G(\mathbf{x}, \mathbf{y})=g(\vartheta(\mathbf{x}, \mathbf{y}))$ for all $\mathbf{x}, \mathbf{y} \in \mathbb{S}^{d}$, where $\vartheta(\mathbf{x}, \mathbf{y})$ is the spherical distance between $\mathbf{x}$ and $\mathbf{y}$ defined in (1.0.3).

Definition 2.1.7. (i) A function $G: \mathbb{S}^{d} \times \mathbb{S}^{d} \rightarrow \mathbb{R}$ is said to be positive definite on $\mathbb{S}^{d}$, if the inequality

$$
\sum_{i=1}^{n} \sum_{j=1}^{n} a_{i} a_{j} G\left(\mathbf{x}_{i}, \mathbf{x}_{j}\right) \geq 0
$$

holds for every $n \in \mathbb{N}$, any $\mathbf{a}_{i} \in \mathbb{R}$ and $\mathbf{x}_{i} \in \mathbb{S}^{d}(i=1, \cdots, n)$.

(ii) A function $g\left(\vartheta\left(\mathbf{x}_{1}, \mathbf{x}_{2}\right)\right)$, or $g(\vartheta)$ for simplicity, is called an isotropic positive definite function on $\mathbb{S}^{d}$ if

$$
\sum_{i=1}^{n} \sum_{j=1}^{n} a_{i} a_{j} g\left(\vartheta\left(\mathbf{x}_{i}, \mathbf{x}_{j}\right)\right) \geq 0
$$

holds for every $n \in \mathbb{N}$, any $a_{i} \in \mathbb{R}$ and $\mathbf{x}_{i} \in \mathbb{S}^{d}, i=1, \ldots, n$.

\subsection{The Gamma Function}

The Euler integral of the second kind

$$
\Gamma(z)=\int_{0}^{\infty} x^{z-1} e^{-x} d x
$$

defines the Gamma function $\Gamma(z)$ for all complex numbers which have the positive real part. In particular, $\Gamma(1)=1$. Using integration by parts, we get the recurrence formula

$$
\Gamma(z)=-\left[x^{z-1} e^{-x}\right]_{0}^{\infty}+(z-1) \int_{0}^{\infty} x^{z-2} e^{-x} d x=(z-1) \Gamma(z-1) .
$$

If we restrict the domain on the set of positive integers $\mathbb{N}$, then we get

$$
\Gamma(n)=(n-1) !
$$

Now, we list the following lemma [19] as a reference.

Lemma 2.2.1. If $n \rightarrow \infty$, then

$$
\frac{\Gamma(n+k)}{\Gamma(n)} \sim n^{k}, \quad k \in \mathbb{R},
$$

where the symbol $\sim$ means that the ratio of the two sides converges to 1 or asymptotically converges. 


\subsection{Orthogonal Polynomial}

Gegenbauer polynomials or ultraspherical polynomials, denoted by $P_{n}^{(\alpha)}(x)$, are orthogonal polynomials on the interval $[-1,1]$ with respect to the weight function $\left(1-x^{2}\right)^{\alpha-\frac{1}{2}}$. They are special cases of Jacobi polynomials, occasionally called hypergeometric polynomials, denoted by $P_{n}^{(\alpha, \beta)}(x)$, which are a class of classical orthogonal polynomials. They are orthogonal with respect to the weight $(1-x)^{\alpha}(1-x)^{\beta}$ on the interval $[-1,1]$.

The Gegenbauer polynomials $P_{n}^{(\alpha)}(x)$ of degree $n$ can be defined in terms of their generating function [9]:

$$
\frac{1}{\left(1-2 x t+t^{2}\right)^{\alpha}}=\sum_{n=0}^{\infty} P_{n}^{(\alpha)}(x) t^{n}, \quad t \in(-1,1)
$$

The function $P_{n}^{(\alpha)}(x)$ is even if $n$ is even and odd if $n$ is odd. For $\alpha=0$, we follow [22] and set

$$
P_{n}^{(0)}(\cos \vartheta)=\cos n \vartheta, \quad n \in \mathbb{N}_{0}, \vartheta \in[0, \pi]
$$

The Gegenbauer polynomials satisfy the recurrence relation [24]:

$$
\begin{aligned}
& P_{(0)}^{\alpha}(x)=1 \\
& P_{(1)}^{\alpha}(x)=2 \alpha x \\
& P_{(n)}^{\alpha}(x)=\frac{1}{n}\left[2 x(n+\alpha-1) P_{n-1}^{\alpha}(x)-(n+2 \alpha-2) P_{n-2}^{\alpha}(x)\right], \quad n \in \mathbb{N}_{0} .
\end{aligned}
$$

The Jacobi polynomials are defined as follows:

$$
P_{n}^{(\alpha, \beta)}(x)=\frac{(\alpha+1)_{n}}{n !}{ }_{2} F_{1}\left(-n, 1+\alpha+\beta+n ; \alpha+1 ; \frac{1}{2}(1-x)\right),
$$

where

$$
{ }_{2} F_{1}(a, b ; c ; z)=\sum_{n=0}^{\infty} \frac{(a)_{n}(b)_{n}}{(c)_{n}} \frac{z^{n}}{n !}
$$

is the hypergeometric function which is defined for $|z|<1$ by the power series and $(\alpha+1)_{n}$ denotes the Pochhammer's symbol (for the rising factorial) ,i.e.,

$$
(\alpha+1)_{n}=(\alpha+1)(\alpha+2), \cdots,(\alpha+n+1)
$$


In this case, the series for the hypergeometric function is finite, and an explicit expression [25] of $P_{n}^{(\alpha, \beta)}(x)$ is

$$
P_{n}^{(\alpha, \beta)}(x)=\sum_{m=0}^{n} \frac{\Gamma(\alpha+n+1)}{n ! \Gamma(\alpha+\beta+n+1)}\left(\begin{array}{c}
n \\
m
\end{array}\right) \frac{\Gamma(\alpha+\beta+n+m+1)}{\Gamma(\alpha+m+1)}\left(\frac{x-1}{2}\right)^{m}, n \in N_{0} .
$$

For reference later on, we note the identities of Jacobi polynomials

$$
\begin{gathered}
P_{n}^{(\alpha, \beta)}(1)=\left(\begin{array}{c}
n+\alpha \\
n
\end{array}\right), \\
P_{0}^{(\alpha, \beta)}(x)=1 .
\end{gathered}
$$

If $\alpha=\beta$, the Jacobi polynomial $P_{n}^{(\alpha, \beta)}(x)$ reduces to the Gegenbauer polynomial, with

$$
P_{n}^{\left(\alpha-\frac{1}{2}, \alpha-\frac{1}{2}\right)}(x)=\frac{\Gamma(2 \alpha) \Gamma\left(n+\alpha+\frac{1}{2}\right)}{\Gamma\left(\alpha+\frac{1}{2}\right) \Gamma(n+2 \alpha)} P_{n}^{(\alpha)}(x), \quad n \in \mathbb{N}_{0}, \quad \alpha>-\frac{1}{2} .
$$

\subsection{Bessel Functions}

The Bessel function of the first kind of order $\alpha$, denoted as $J_{\alpha}(z)$, is defined by its series expansion around $z=0$,

$$
J_{\alpha}(z)=\sum_{\nu}^{\infty} \frac{(-1)^{\nu}(z / 2)^{\alpha+2 \nu}}{\nu ! \Gamma(\nu+\alpha+1)} .
$$

where $\Gamma(x)$ is the Gamma function, $\alpha$ is arbitrary real.

Bessel functions are solutions of Bessel's differential equation

$$
x^{2} \frac{d^{2} y}{d x^{2}}+x \frac{d y}{d x}+\left(x^{2}-\alpha^{2}\right) y=0
$$

If $\alpha=-\frac{1}{2}$

$$
J_{-\frac{1}{2}}(z)=\left(\frac{2}{\pi z}\right)^{\frac{1}{2}} \cos z
$$

If $\alpha=\frac{1}{2}$,

$$
J_{\frac{1}{2}}(z)=\left(\frac{2}{\pi z}\right)^{\frac{1}{2}} \sin z
$$

Bessel functions have the following asymptotic forms, when $\alpha$ is fixed and $z \rightarrow 0$,

$$
\begin{gathered}
J_{\alpha}(z) \rightarrow 1 \\
J_{\alpha}(z) \sim \frac{\left(\frac{z}{2}\right)^{\frac{1}{2}}}{\Gamma(\alpha+1)} .
\end{gathered}
$$


The integral representation

$$
J_{\alpha}(z)=\frac{\left(\frac{z}{2}\right)^{\frac{1}{2}}}{\Gamma\left(\alpha+\frac{1}{2}\right) \Gamma\left(\frac{1}{2}\right)} \int_{-1}^{1}\left(1-x^{2}\right)^{\left(\alpha-\frac{1}{2}\right)} e^{i z x} d x
$$

holds for $\alpha>-\frac{1}{2}$.

\subsection{Abel-Summability}

In order to use the Abel-summability in the proof of the convergence of the series $\sum_{n=0}^{\infty} b_{n} P_{n}^{\frac{d-1}{2}}(1)$ in Chapter 4 and Chapter 5, we state Abel-summability and Abel theorem here.

Abel-summability is a generalized convergence criterion for power series. It extends the usual definition of the sum of a series, and gives a way of summing up certain divergent series. Let us start with a series $\sum_{n=0}^{\infty} c_{n}$, convergent or not, and use that series to define a power series $f(x)=\sum_{n=0}^{\infty} c_{n} x^{n}$. Note that for $|x|<1$ the summability of $f(x)$ is easier to achieve than the summability of the original series. Starting with this observation we say that the series $\sum_{n=0}^{\infty} c_{n}$ is Abel-summable if the defining series $f(x)$ is convergent for all $|x|<1$, and if $f(x)$ converges to some limit $s$ as $x \rightarrow 1^{-}$. If this is so, we shall say that $\sum_{n=0}^{\infty} c_{n}$ Abel converges to $s$. In other words, If

$$
\lim _{x \rightarrow 1^{-}} \sum_{n=0}^{\infty} c_{n} x^{n}=s
$$

then

$$
\sum_{n=0}^{\infty} c_{n}=s
$$

Of course it is important to ask whether an ordinary convergent series is also Abesummable, and whether it converges to the same limit? This is true, and the result is known as Abel's limit theorem, or simply as Abel's theorem [3].

Theorem 2.5.1 (Abel's Theorem). Let

$$
f(x)=\sum_{n=0}^{\infty} c_{n} x^{n}
$$


be a power series with real coefficients $c_{n}$ and radius of convergence 1 . Suppose that the series

$$
\sum_{n=0}^{\infty} c_{n}
$$

is convergent. Then $f(x)$ is continuous from the left at $x=1$, i.e.

$$
\lim _{x \rightarrow 1^{-}} \sum_{n=0}^{\infty} c_{n} x^{n}=\sum_{n=0}^{\infty} c_{n} .
$$




\section{CHAPTER 3}

\section{ISOTROPIC POSITIVE DEFINITE FUNCTIONS IN EUCLIDEAN SPACE}

This chapter reviews some properties of isotropic definite functions in Euclidean space

$\mathbb{R}^{d}$. The main result is the first inequality in the abstract which is the key to prove the positive definiteness of an isotropic function on spheres $\mathbb{S}^{d}$.

\subsection{Integral Transforms}

Before getting into the discussion of the characterizations of positive definite functions we summarize some formulas for various integral transforms to be used later.

We start with the conventions of the Fourier transform, for which a reference book is Bochner and Chandrasekhara [5].

Definition 3.1.1. The Fourier transform of $f \in L^{1}\left(\mathbb{R}^{d}\right)$ is given by

$$
\hat{g}(\mathbf{u})=\int_{\mathbb{R}^{d}} g(\mathbf{x}) e^{i \mathbf{u}^{\prime} \mathbf{x}} d \mathbf{x}, \quad \mathbf{u} \in \mathbb{R}^{d}
$$

and its inverse Fourier transform is given by

$$
\check{g}(\mathbf{x})=\frac{1}{(2 \pi)^{d}} \int_{\mathbb{R}^{d}} g(\mathbf{u}) e^{-i \mathbf{x}^{\prime} \mathbf{u}} d \mathbf{u}, \quad \mathbf{x} \in \mathbb{R}^{d}
$$

where $\mathbf{x}^{\prime} \mathbf{u}$ is the inner product between $\mathbf{x}$ and $\mathbf{u}$.

In this thesis, we will focus on isotropic positive definite functions. Note that the Fourier transform of an isotropic function is again isotropic [26]. Indeed, the Fourier transform of an isotropic function is its own inverse.

Theorem 3.1.2. Let $G \in L_{1}\left(\mathbb{R}^{d}\right)$ be continuous and isotropic, i.e, $G(\mathbf{x})=g(\|\mathbf{x}\|)$. Then its Fourier transform is also isotropic, i.e, $\hat{G}$ is also isotropic, i.e, $\hat{G}(\mathbf{x})=\mathcal{F}_{d} g(\|\mathbf{x}\|)$ with

$$
\mathcal{F}_{d} g(r)=(2 \pi)^{\frac{d}{2}} x^{-\frac{d-2}{2}} \int_{0}^{\infty} g(u) u^{\frac{d}{2}} J_{\frac{d-2}{2}}(x u) d u,
$$

where $r=\|\mathbf{x}\|$ and $J_{\frac{d-2}{2}}(x)$ is the classical Bessel function of the first kind of order $\frac{d-2}{2}$. 
Remark 1: The integral transform in (3.1.1) is also referred to as a Fourier-Bessel transform or Hankel transform.

Remark 2: The Hankel inversion theorem [23] ensures that the Fourier transform for an isotropic function is its own inverse, i.e., for an isotropic function $g$,

$$
\mathcal{F}_{d}\left[\mathcal{F}_{d} g\right]=g
$$

\subsection{Characterizations of Positive Definite Functions}

One of the most celebrated results on positive definite function in Euclidean space $\mathbb{R}^{d}$ is Bochner's integral characterization, which is established by Bochner in 1932 for $d=1$ and 1933 for $d>1$.

The following theorem established the identity of positive definite functions in $\mathbb{R}^{d}$ with characteristic functions of probability measures in $\mathbb{R}^{d}$.

Theorem 3.2.1 (Bochner's Theorem). A complex-valued function $g \in C\left(\mathbb{R}^{d}\right)$ is positive definite in $\mathbb{R}^{d}$ if and only if it is the Fourier transform of a finite nonnegative Borel measure $\nu$ on $\mathbb{R}^{d}$, i.e.,

$$
g(\mathbf{x})=\int_{\mathbb{R}^{d}} e^{i \mathbf{x}^{\prime} \mathbf{y}} \nu(d \mathbf{y}), \quad \mathbf{x} \in \mathbb{R}^{d} .
$$

Remark: This is the Bochner's integral characterization for the positive definite functions in $\mathbb{R}^{d}$. One of the proof is given by Wendland [27]. The proof for $d=1$ can be found in the probability textbook [7].

From Bochner's Theorem, an isotropic positive definite function $g$ in $\mathbb{R}^{d}$ is the Fourier transform of a finite nonnegative Borel measure $\nu$ on $\mathbb{R}^{d}$. Then the inverse Fourier transform of an isotropic positive definite function $g$ is a finite nonnegative Borel measure $\nu$ on $\mathbb{R}^{d}$, so it is nonnegative. From (3.1.2), the inverse Fourier transform for an isotropic function is its Fourier transform. Therefore, the inverse Fourier transform or Fourier transform of an isotropic positive definite function is nonnegative. The Fourier transform of an isotopic 
positive definite function is given by (3.1.1). Now we obtain,

$$
(2 \pi)^{\frac{d}{2}} x^{-\frac{d-2}{2}} \int_{0}^{\infty} g(u) u^{\frac{d}{2}} J_{\frac{d-2}{2}}(x u) d u \geq 0, \quad x \geq 0
$$

or, simplify,

$$
\int_{0}^{\infty} g(u) u^{\frac{d}{2}} J_{\frac{d-2}{2}}(x u) d u \geq 0, \quad x \geq 0 .
$$

\subsection{Schoenberg's Characterization}

A well-known result on isotropic positive definite functions in Euclidean space $\mathbb{R}^{d}$ is Schoenberg's characterization [21] established in 1938. It is stated as the following theorem $[10]$

Theorem 3.3.1. A continuous function $g(\|\mathbf{x}\|): \mathbb{R}^{d} \rightarrow \mathbb{R}$ is isotropic positive definite if and only if $g(x)$ is the Bessel transform of a finite nonnegative Borel measure $\nu$ on $[0, \infty)$, i.e.,

$$
g(x)=\int_{0}^{\infty} \Omega_{d}(x t) d \nu(t)
$$

where

$$
\Omega_{d}(x)= \begin{cases}\cos x & d=1 \\ \Gamma\left(\frac{d}{2}\right)\left(\frac{2}{r}\right)^{\frac{d-2}{2}} J_{\frac{d-2}{2}}(x) & d \geq 2\end{cases}
$$

is an integral transcendental function and $J_{\frac{d-2}{2}}(x)$ is the Bessel function of the first kind of $\operatorname{order} \frac{d-2}{2}$.

If a function is isotropic positive definite in $\mathbb{R}^{d}$, then it is also isotropic positive definite in $\mathbb{R}^{d-1}$. Therefore, we are interested in those functions which are isotropic positive definite in $\mathbb{R}^{d}$ for all $d \in \mathbb{N}$. Those functions are identified by the following theorem.

Theorem 3.3.2. A continuous function $g:[0, \infty) \rightarrow \mathbb{R}$ makes $g(\|\mathbf{x}\|)$ an isotropic positive definite function in all $\mathbb{R}^{d}$ if and only if it is of the form

$$
g(x)=\int_{0}^{\infty} e^{-x^{2} t^{2}} d \nu(t)
$$

where $\nu$ is a finite nonnegative Borel measure on $[0, \infty)$. 


\subsection{Properties of Positive Definite Functions}

In order to derive the properties of positive definite functions easily, we have expanded the range of positive definite functions on the set of complex numbers in Definition 2.1.2.

The reader should note that we call a function positive definite if the left side of (2.1.1) is nonnegative and strictly positive definite if the left side of (2.1.1) is positive for $z \neq 0$. This seems to be natural. However, there is an alternative terminology around in the literature: some authors call a function positive semi-definite if the left side of (2.1.1) is nonnegative and positive definite if if the left side of (2.1.1) is positive for $z \neq 0$. We do not follow this approach here, but the reader should always keep this in mind when looking at other texts.

From Definition 2.1.2, we can read off the elementary properties [27] of such a positive definite function.

Theorem 3.4.1. Suppose $g$ is a complex-valued positive definite function in $\mathbb{R}^{d}$. Then the following properties are satisfied.

(a) $g(\mathbf{0}) \geq 0$.

(b) $g(\mathbf{x})=\overline{g(-\mathbf{x})}$ for all $\mathbf{x} \in \mathbb{R}^{d}$.

(c) $|g(\mathbf{x})| \leq g(\mathbf{0})$ for all $\mathbf{x} \in \mathbb{R}^{d}$, i.e. $g(\mathbf{x})$ is bounded

(d) $g(\mathbf{0})=0$ if and only if $g \equiv 0$.

(e) For $d=1, g$ is continuous at $x=0$ implies that it is uniformly continuous on $\mathbb{R}$.

(f) For $d=1, g$ is uniformly continuous in $\mathbb{R}$, we have for every continuous complexvalued function $\zeta$ on $\mathbb{R}$ and every $T>0$ :

$$
\int_{0}^{T} \int_{0}^{T} g(s-t) \zeta(s) \overline{\zeta(t)} d s d t \geq 0
$$

(g) If $c_{1}, \cdots, c_{m}$ are nonnegative, and $g_{1}, \cdots, g_{m}$ are positive definite, then $G:=\sum_{l=1}^{m} c_{l} g_{l}$ is also positive definite. If one of $g_{l}$ is strictly positive definite and the corresponding $c_{l}$ is positive then $G$ also is strictly positive definite.

(h) For any $a \in \mathbb{R}, f(x)=g(\mathbf{x}) e^{i a \mathbf{x}}$ is also positive definite. Generally, the product of two positive definite functions is positive. 
(i) If $\left\{g_{n}(\mathbf{x})\right\}$ be a sequence of positive definite in $\mathbb{R}^{d}, g_{n}(\mathbf{x}) \rightarrow g(\mathbf{x})$ as $n \rightarrow \infty$, and $g(\mathbf{x})$ is continuous, then $g(\mathbf{x})$ is also a positive definite function in $\mathbb{R}^{d}$.

Proof. (a) Assume that $n=1$ and $z_{1}=1$. By (2.1.1), we obtain

$$
g(\mathbf{0}) \geq 0
$$

(b) Assume that $n=2$. By (2.1.1), we have

$$
g(\mathbf{0})\left|z_{1}\right|^{2}+g\left(\mathbf{x}_{1}-\mathbf{x}_{2}\right) z_{1} \overline{z_{2}}+g\left(\mathbf{x}_{2}-\mathbf{x}_{1}\right) z_{2} \overline{z_{1}}+g(\mathbf{0})\left|z_{2}\right|^{2} \geq 0
$$

Taking $x_{1}=\mathbf{0}, x_{2}=\mathbf{x}, z_{1}=z_{2}=i$ in (3.4.2), we have

$$
2 g(\mathbf{0})+g(-\mathbf{x})+g(\mathbf{x}) \geq 0
$$

which implies $g(-\mathbf{x})+g(\mathbf{x})$ is real.

Taking $x_{1}=\mathbf{0}, x_{2}=\mathbf{x}, z_{1}=i, z_{2}=1$ in (3.4.2), we have

$$
2 g(\mathbf{0})+i g(-\mathbf{x})-i g(\mathbf{x}) \geq 0
$$

which implies $g(-\mathbf{x})-g(\mathbf{x})$ is pure imaginary. Hence, we have

$$
g(\mathbf{x})=\overline{g(-\mathbf{x})}
$$

(c) Taking $x_{1}=\mathbf{0}, x_{2}=\mathbf{x}, z_{1}=g(\mathbf{x}), z_{2}=-|g(\mathbf{x})|$ in (3.4.2), we have

$$
g(\mathbf{0})|g(\mathbf{x})|^{2}+g(-\mathbf{x}) g(\mathbf{x})(\overline{-|g(\mathbf{x})|})+g(\mathbf{x})(-|g(\mathbf{x})|) \overline{g(\mathbf{x})}+g(\mathbf{0})|g(\mathbf{x})|^{2} \geq 0
$$

By (b), we have

$$
\begin{aligned}
& g(\mathbf{0})|g(\mathbf{x})|^{2}+\overline{g(\mathbf{x})} g(\mathbf{x})(\overline{-|g(\mathbf{x})|})+g(\mathbf{x})(-|g(\mathbf{x})|) \overline{g(\mathbf{x})}+g(\mathbf{0})|g(\mathbf{x})|^{2} \\
= & 2 g(\mathbf{0})|g(\mathbf{x})|^{2}+2 g(-\mathbf{x}) g(\mathbf{x})(\overline{-|g(\mathbf{x})|}) \\
= & 2|g(\mathbf{x})|^{2}(g(\mathbf{0})-|g(\mathbf{x})|) \geq 0
\end{aligned}
$$

Therefore,

$$
g(\mathbf{0}) \geq|g(\mathbf{x})|
$$


(d) For the " only if" part,$g(\mathbf{0})=0$ implies $|g(\mathbf{x})|=0$ for all $\mathbf{x} \in \mathbb{R}^{d}$ by (c). The converse is obvious.

(e) From (2.1.1) and (b), we have that $\left[g\left(x_{j}-x_{k}\right)\right]_{j, k=1}^{n}$ is a Hermitian positive definite matrix. In particular, for $n=3, x_{1}=t, x_{2}=s$ and $t_{3}=0$, the matrix

$$
\left[\begin{array}{ccc}
g(0) & g(t-s) & g(t) \\
\overline{g(t-s)} & g(0) & g(s) \\
g(t) & \overline{g(s)} & g(0)
\end{array}\right]
$$

must have a nonnegative determinant, which gives

$$
\begin{aligned}
0 & \leq g(0)^{3}+g(s) g(t-s) \overline{g(t)}+g(t) \overline{g(s) g(t-s)}-g(0)\left(|g(t)|^{2}+|g(s)|^{2}+|g(t-s)|^{2}\right) \\
& =g(0)^{3}+2 \operatorname{Re}[g(s) g(t-s) \overline{g(t)}]-g(0)\left(|g(t)-g(s)|^{2}+2 \operatorname{Re}[g(s) \overline{g(t)}]+|g(t-s)|^{2}\right) \\
& =g(0)^{3}+2 \operatorname{Re}\left[g(s) \overline{g(t)}(g(t-s)-g(0)]-g(0)\left(|g(t)-g(s)|^{2}+|g(t-s)|^{2}\right)\right. \\
& \leq g(0)^{3}+2 g(0)^{2}|g(0)-g(t-s)|-g(0)\left(|g(t)-g(s)|^{2}+|g(t-s)|^{2}\right)
\end{aligned}
$$

where in the last inequality we used (c). After simplifying and adding $|g(t)-g(s)|^{2}$ to both sides of the inequality we have

$$
\begin{aligned}
|g(t)-g(s)|^{2} & \leq g(0)^{2}-|g(t-s)|^{2}+2 g(0)|g(0)-g(t-s)| \\
& =(g(0)-|g(t-s)|)(g(0)+|g(t-s)|)+2 g(0)|g(0)-g(t-s)| \\
& \leq(g(0)-|g(t-s)|)(g(0)+g(0))+2 g(0)|g(0)-g(t-s)| \\
& \leq 4 g(0)|g(0)-| g(t-s) \mid
\end{aligned}
$$

Thus the "modulus of continuity" at each $t$ is bounded by twice the square root of the that at 0 , and so the continuity at 0 implies uniform continuity everywhere on $\mathbb{R}$.

(f) The integrand of the double integral in (3.4.1) is continuous and the integral is the limit of the Riemann sums, hence it is nonnegative by (2.1.1), i.e.

$$
\int_{0}^{T} \int_{0}^{T} g(s-t) \zeta(s) \overline{\zeta(t)} \geq 0
$$


(g) Since $G:=c_{l} g_{l}$, we have

$$
\begin{aligned}
& \sum_{j=1}^{n} \sum_{k=1}^{n} G\left(\mathbf{x}_{j}-\mathbf{x}_{k}\right) z_{j} \bar{z}_{k} \\
= & \sum_{j=1}^{n} \sum_{k=1}^{n}\left(\sum_{l=1}^{m} c_{l} g_{l}\left(\mathbf{x}_{j}-\mathbf{x}_{k}\right)\right) z_{j} \bar{z}_{k} \\
= & \sum_{l=1}^{m} \sum_{j=1}^{n} \sum_{k=1}^{n} c_{l} g_{l}\left(\mathbf{x}_{j}-\mathbf{x}_{k}\right) z_{j} \bar{z}_{k} \\
= & \sum_{l=1}^{m} c_{l}\left(\sum_{j=1}^{n} \sum_{k=1}^{n} g_{l}\left(x_{j}-x_{k}\right) z_{j} \bar{z}_{k}\right) \\
\geq & 0
\end{aligned}
$$

where in the last line we used $c_{l} \geq 0$ and the positive definiteness of $g_{l}$ for $1=1,2, \cdots, m$. If one of $g_{l}$ is strictly positive definite and the corresponding $c_{l}$ is positive, without loss of generality, let $l=p(0 \leq p \leq m)$, then we have

$$
\left.c_{p} \sum_{j=1}^{n} \sum_{k=1}^{n} g_{p}\left(\mathbf{x}_{j}-\mathbf{x}_{k}\right) z_{j} \bar{z}_{k}\right)>0,
$$

hence,

$$
\sum_{l=1}^{m} c_{l}\left(\sum_{j=1}^{n} \sum_{k=1}^{n} g_{l}\left(\mathbf{x}_{j}-\mathbf{x}_{k}\right) z_{j} \bar{z}_{k}\right)>0 .
$$

By Definition 2.1.2, $G$ is strictly positive definite.

(h) Changing $g$ to $f$ in (2.1.1), we have

$$
\begin{aligned}
& \sum_{j=1}^{n} \sum_{k=1}^{n} f\left(\mathbf{x}_{j}-\mathbf{x}_{k}\right) z_{j} \overline{z_{k}} \\
= & \sum_{j=1}^{n} \sum_{k=1}^{n} g\left(\mathbf{x}_{j}-\mathbf{x}_{k}\right) e^{i a\left(\mathbf{x}_{j}-\mathbf{x}_{k}\right)} z_{j} \overline{z_{k}} \\
= & \sum_{l=1}^{m} \sum_{j=1}^{n} g\left(\mathbf{x}_{j}-\mathbf{x}_{k}\right) e^{i a \mathbf{x}_{j}} e^{-i a \mathbf{x}_{j}} z_{j} \overline{z_{k}} \\
= & \sum_{l=1}^{m} \sum_{j=1}^{n} g\left(\mathbf{x}_{j}-\mathbf{x}_{k}\right) e^{i a \mathbf{x}_{j}} e^{\overline{i a \mathbf{x}_{j}}} z_{j} \overline{z_{k}} \\
= & \sum_{l=1}^{m} \sum_{j=1}^{n} g\left(\mathbf{x}_{j}-\mathbf{x}_{k}\right) e^{i a \mathbf{x}_{j}} z_{j} \overline{e^{i a \mathbf{x}_{j}} z_{k}} \\
\geq & 0
\end{aligned}
$$


where the inequality is because of the positive definiteness of $g$. By the definition, $f(x)=$ $g(x) e^{i a \mathrm{x}}$ is also positive definite. The proof for the general case can be found in [27].

(i) Since $\left\{g_{n}(\mathbf{x})\right\}$ be a sequence of positive definite in $\mathbb{R}^{d}, g_{n}(\mathbf{x}) \rightarrow g(\mathbf{x})$ as $n \rightarrow \infty$, and $g(x)$ is continuous, we have

$$
\begin{aligned}
& \sum_{j=1}^{n} \sum_{k=1}^{n} g\left(\mathbf{x}_{j}-\mathbf{x}_{k}\right) z_{j} \overline{z_{k}} \\
= & \sum_{j=1}^{n} \sum_{k=1}^{n} \lim _{i \rightarrow \infty} g_{i}\left(\mathbf{x}_{j}-\mathbf{x}_{k}\right) z_{j} \overline{z_{k}} \\
= & \lim _{i \rightarrow \infty} \sum_{j=1}^{n} \sum_{k=1}^{n} g_{i}\left(\mathbf{x}_{j}-\mathbf{x}_{k}\right) z_{j} \overline{z_{k}} \\
\geq & 0 .
\end{aligned}
$$

By Definition 2.1.2, $g(\mathbf{x})$ is also positive definite in $\mathbb{R}^{d}$. 


\section{CHAPTER 4}

\section{ISOTROPIC POSITIVE DEFINITE FUNCTIONS ON SPHERES}

In this chapter, we proceed to review Schogenberg's characterizations and constructions of isotropic positive definite functions on $\mathbb{S}^{d}$ in terms of Gegenbauer polynomials, for which we require some classical results concerning Gegenbauer polynomials.

\subsection{Some Classical Results Concerning Ultraspherical Polynomials}

We introduce a representation of a real continuous function by Gegenbauer polynomials $[22]$.

Lemma 4.1.1. A real continuous function $G(\mathbf{x})$ defined on $\mathbb{S}^{d}$ can be approximated by

$$
G(\mathbf{x}) \sim \sum_{n=0}^{\infty} \frac{n+\alpha}{\alpha \omega_{d}} \int_{\mathbb{S}_{d}} G(\mathbf{y}) P_{n}^{(\alpha)}(\cos \theta) d \omega_{\mathbf{y}}
$$

where $\alpha=\frac{d-1}{2}, \omega_{d}=\frac{2 \pi \frac{d+1}{2}}{\Gamma\left(\frac{d+1}{2}\right)}$ is the surface area of $\mathbb{S}^{d}$ and $\theta$ is the spherical distance between $\mathbf{x}$ and $\mathbf{y}$.

Proof. Suppose that $G(\mathbf{x})$ is a real continuous function defined on $\mathbb{S}^{d}$. Let $\mathbf{x}_{r}$ be a point on the radius ox such that $\mathbf{o x}_{r}=r, 0 \leq r<1$. Define $G\left(\mathbf{x}_{r}\right)$ as a Poisson integral

$$
G\left(\mathbf{x}_{r}\right)=\frac{1}{\omega_{d}} \int_{\mathbb{S}_{d}} \frac{1-r^{2}}{\left(1-2 r \cos \theta+r^{2}\right)^{\frac{d+1}{2}}} G(\mathbf{y}) d \omega_{\mathbf{y}} .
$$

By Proposition 2.3.2 in [14], we have that

$$
\lim _{r \rightarrow 1} G\left(\mathbf{x}_{r}\right)=G(\mathbf{x})
$$

Let $t=r$ and $x=\cos \theta$ in (2.3.1), we obtain

$$
\frac{1}{\left(1-2 r \cos \theta+r^{2}\right)^{\alpha}}=\sum_{n=0}^{\infty} r^{n} P_{n}^{(\alpha)}(\cos \theta) .
$$

Differentiating both sides of (4.1.5) with respect to $r$, we get

$$
\frac{-2 \cos \theta+2 r}{\left(1-2 r \cos \theta+r^{2}\right)^{\alpha+1}}=\sum_{n=0}^{\infty} n r^{n-1} P_{n}^{(\alpha)}(\cos \theta)
$$


Multiplying (4.1.5) by $\frac{r}{\alpha}$, we have

$$
\frac{-2 r \cos \theta+2 r^{2}}{\alpha\left(1-2 r \cos \theta+r^{2}\right)^{\alpha+1}}=\frac{1}{\alpha} \sum_{n=0}^{\infty} n r^{n} P_{n}^{(\alpha)}(\cos \theta) .
$$

Adding (4.1.4) and (4.1.6), we now have

$$
\frac{1-r^{2}}{\left(1-2 r \cos \theta+r^{2}\right)^{\alpha+1}}=\sum_{n=0}^{\infty}\left(\frac{n}{\alpha}+1\right) r^{n} P_{n}^{(\alpha)}(\cos \theta) .
$$

Multiplying (4.1.7) by $G(\mathbf{y}) \frac{d \omega_{\mathbf{y}}}{\omega_{d}}$ and integrating both sides over $\mathbb{S}^{d}$, we have

$$
\frac{1}{\omega_{d}} \int_{\mathbb{S}_{d}} \frac{1-r^{2}}{\left(1-2 r \cos \theta+r^{2}\right)^{\frac{d+1}{2}}} G(\mathbf{y}) d \omega_{\mathbf{y}}=\int_{\mathbb{S}_{d}} \sum_{n=0}^{\infty} \frac{n+\alpha}{\alpha \omega_{d}} r^{n} G(\mathbf{y}) P_{n}^{(\alpha)}(\cos \theta) d \omega_{\mathbf{y}} .
$$

As $r \rightarrow 1$ in (4.1.8), we develop an approximation of $G(\mathbf{x})$ by

$$
G(\mathbf{x}) \sim \sum_{n=0}^{\infty} \frac{n+\alpha}{\alpha \omega_{d}} \int_{\mathbb{S}_{d}} G(\mathbf{y}) P_{n}^{(\alpha)}(\cos \theta) d \omega_{\mathbf{y}}
$$

The relation (4.1.3) implies that the approximation (4.1.9) is Abel-summable at any point $\mathbf{x}$ on $\mathbb{S}^{d}$ to the sum $G(\mathbf{x})$.

We may convert the Cartesian coordinates of $\mathbf{x}=\left(x, x_{1}, \cdots, x_{d}\right) \in \mathbb{R}^{d+1}$ to spherical coordinates with:

$$
\begin{aligned}
x & =\cos \vartheta \\
x_{1} & =\sin \vartheta \cos \vartheta_{1}, \\
x_{2} & =\sin \vartheta \sin \vartheta_{1} \cos \vartheta_{2}, \\
& \vdots \\
x_{d-2} & =\sin \vartheta \sin \vartheta_{1} \cdots \cos \vartheta_{d-2}, \\
x_{d-1} & =\sin \vartheta \sin \vartheta_{1} \cdots \sin \vartheta_{d-2} \cos \phi, \\
x_{d} & =\sin \vartheta \sin \vartheta_{1} \cdots \sin \vartheta_{d-2} \sin \phi,
\end{aligned}
$$

where the angles $\vartheta, \vartheta_{1}, \cdots, \vartheta_{d-2} \in[0, \pi]$, and $\phi \in[0,2 \pi)$.

Let $G(\mathbf{x})=g(\vartheta)$ and $\alpha=\frac{d-1}{2}$. The integral in (4.1.9) can be written

$$
A_{n}=\int_{0}^{\pi} \cdots \int_{0}^{\pi} \int_{0}^{2 \pi} g\left(\vartheta^{\prime}\right) P_{n}^{(\alpha)}(\cos \theta) \sin ^{d-1} \vartheta^{\prime} \sin ^{d-2} \vartheta_{1}^{\prime} \cdots \sin \vartheta_{d-2}^{\prime} d \vartheta^{\prime} \cdots d \vartheta_{d-2}^{\prime} d \phi^{\prime}
$$


where $\mathbf{y}=\left(x^{\prime}, x_{1}^{\prime}, \cdots, x_{d}^{\prime}\right)$ in terms of Euclidean coordinate and $\mathbf{y}=\left(\vartheta^{\prime}, \vartheta_{1}^{\prime}, \cdots, \vartheta_{d-2}^{\prime}, \phi^{\prime}\right)$ in terms of spherical coordinates. Since the integrand $g\left(\vartheta^{\prime}\right) P_{n}^{(\alpha)}(\cos \theta) \sin ^{d-1} \vartheta^{\prime}$ only involves in $\vartheta^{\prime}$, we may integrate first with the variable $\vartheta_{1}^{\prime}, \cdots, \vartheta_{d-2}^{\prime}, \phi^{\prime}$ as follows:

$$
A_{n}=\int_{0}^{\pi} g\left(\vartheta^{\prime}\right) C_{n} \sin ^{d-1} \vartheta^{\prime} d \vartheta^{\prime}
$$

where

$$
C_{n}=\int_{0}^{\pi} \cdots \int_{0}^{\pi} \int_{0}^{2 \pi} P_{n}^{(\alpha)}(\cos \theta) \sin ^{d-2} \vartheta_{1}^{\prime} \cdots \sin \vartheta_{d-2}^{\prime} d \vartheta^{\prime} \cdots d \vartheta_{d-2}^{\prime} d \phi^{\prime} .
$$

This integral can be reduced as follows. For two points $\mathbf{p}, \mathbf{q}$ both lie in the equator $\mathbb{S}^{d-1}$ defined by $\vartheta=\frac{\pi}{2}$ with the polar coordinate:

$$
\mathbf{p}=\left(\frac{\pi}{2}, \vartheta_{1}, \cdots, \vartheta_{d-2}, \phi\right), \quad \mathbf{q}=\left(\frac{\pi}{2}, \vartheta_{1}^{\prime}, \cdots, \vartheta_{d-2}^{\prime}, \phi^{\prime}\right)
$$

Then we get

$$
\cos \theta=\mathbf{x}^{\prime} \mathbf{y}=x x^{\prime}+x_{1} x_{1}^{\prime}+\cdots+x_{d} x_{d}^{\prime}=\cos \vartheta \cos \vartheta^{\prime}+\sin \vartheta \sin \vartheta^{\prime} \cos \psi
$$

where $\psi$ is the spherical distance between $\mathbf{p}$ and $\mathbf{q}$.

Now, $C_{n}$ is an integration of $P_{n}^{(\alpha)}(\cos \theta)$ over $\mathbb{S}^{d}$. If we take in $\mathbb{S}^{d}$ a different polar coordinates $\left(\psi, \psi_{1}, \cdots, \psi_{d-3}, \varphi\right)$ of pole $\mathbf{p}$, we obtain

$$
C_{n}=\int_{0}^{\pi} \cdots \int_{0}^{\pi} \int_{0}^{2 \pi} P_{n}^{(\alpha)}(\cos \theta) \sin ^{d-2} \psi \sin ^{d-3} \psi_{1} \cdots \sin \psi_{d-3} d \psi d \psi_{1} \cdots d \psi_{d-3} d \varphi
$$

Since $\cos \theta$ depends only on $\psi$ by (4.1.13), we have

$$
C_{n}=\omega_{d-2} \int_{0}^{\pi} P_{n}^{(\alpha)}(\cos \theta) \sin ^{d-2} \psi d \psi
$$

which can be explicitly computed by

$$
\int_{0}^{\pi} P_{n}^{(\alpha)}(\cos \theta) \sin ^{2 \alpha-1} \psi d \psi=\frac{\Gamma(\alpha) \Gamma\left(\frac{1}{2}\right) \Gamma(n+1) \Gamma(2 \alpha)}{\Gamma\left(\alpha+\frac{1}{2}\right) \Gamma(n+2 \alpha)} P_{n}^{\alpha}(\cos \vartheta) P_{n}^{\alpha}\left(\cos \vartheta^{\prime}\right)
$$

Substituting $C_{n}$ into (4.1.11), the expression (4.1.9) reduces to

$$
g(\vartheta) \sim \sum_{n=o}^{\infty} \frac{(n+\alpha) \Gamma(\alpha) \Gamma(n+1) \Gamma(2 \alpha)}{\Gamma\left(\alpha+\frac{1}{2}\right) \Gamma\left(\frac{1}{2}\right) \Gamma(n+2 \alpha)} P_{n}^{\alpha}(\cos \vartheta) \int_{0}^{\pi} P_{n}^{\alpha}\left(\cos \vartheta^{\prime}\right) g\left(\vartheta^{\prime}\right) \sin ^{d-1} \vartheta^{\prime} d \vartheta^{\prime}
$$


We note that this expansion is also Abel-summable.

We need the following two lemmas due to Schoenberg to prove the Schoenberg's characterization.

Lemma 4.1.2. If $g(x)$ is a real continuous function on $[-1,1]$ such that $g(\theta)$ is positive definite on $\mathbb{S}^{d}$, then we have

$$
\int_{\mathbb{S}^{d}} g(\theta) d \omega_{\mathbf{y}} \geq 0
$$

where $\theta$ is the spherical distance between $\mathbf{x}$ and $\mathbf{y}$.

Lemma 4.1.3. The Gegenbauer polynomials

$$
P_{n}^{\frac{d-1}{2}}(\cos \vartheta), \quad n \in \mathbb{N}_{0}, \vartheta \in[0, \pi]
$$

are all positive definite on $\mathbb{S}^{d}$.

\subsection{Schoenberg's Characterization}

Theorem 4.2.1 (Schoenberg's Characterization). A real continuous function $g(x)$ on $[0, \pi]$ is positive definite on $\mathbb{S}^{d}$ if and only if $g(\vartheta)$ can be expressed as the series in (4.1.14) with the series converges for $\vartheta=0$ and the coefficients of $P_{n}^{\frac{d-1}{2}}(\cos \vartheta)$ are nonnegative, i.e.

$$
\sum_{n=0}^{\infty} \frac{\left(n+\frac{d-1}{2}\right) \Gamma\left(\frac{d-1}{2}\right) \Gamma(n+1) \Gamma(d-1)}{\Gamma\left(\frac{d}{2}\right) \Gamma\left(\frac{1}{2}\right) \Gamma(n+d-1)} \int_{0}^{\pi} P_{n}^{\frac{d-1}{2}}(\cos \vartheta) g(\vartheta) \sin ^{d-1} \vartheta d \vartheta P_{n}^{\frac{d-1}{2}}
$$

converges, and

$$
\frac{\left(n+\frac{d-1}{2}\right) \Gamma\left(\frac{d-1}{2}\right) \Gamma(n+1) \Gamma(d-1)}{\Gamma\left(\frac{d}{2}\right) \Gamma\left(\frac{1}{2}\right) \Gamma(n+d-1)} \int_{0}^{\pi} P_{n}^{\frac{d-1}{2}}(\cos \vartheta) g(\vartheta) \sin ^{d-1} \vartheta d \vartheta \geq 0,
$$

which is equivalent to

$$
b_{n}=\int_{0}^{\pi} g(\vartheta) P_{n}^{\left(\frac{d-1}{2}\right)}(\cos \vartheta) \sin ^{d-1} \vartheta d \vartheta, \quad n \in \mathbb{N}_{0}
$$

are nonnegative. In other words, $g(\vartheta)$ is positive definite on $\mathbb{S}^{d}$ if and only if

$$
g(\vartheta)=\sum_{n=0}^{\infty} b_{n} P_{n}^{\alpha}(\cos \vartheta)
$$

with $b_{n} \geq 0$ and the series converges for $\vartheta=0$. 
Proof. We can rewrite the integral coefficient of $P_{n}^{\alpha}(\cos \vartheta)$ in (4.1.14) as

$$
D_{n}=\int_{0}^{\pi} P_{n}^{\alpha}\left(\cos \vartheta^{\prime}\right) g\left(\vartheta^{\prime}\right) \sin ^{d-1} \vartheta^{\prime} d \vartheta^{\prime}=\frac{1}{\omega_{d-1}} \int_{\mathbb{S}_{d}} P_{n}^{\alpha}\left(\cos \theta^{\prime}\right) g\left(\theta^{\prime}\right) d \omega_{\mathbf{y}}
$$

where $\theta^{\prime}$ is the spherical distance between $\mathbf{a}=(1,0, \cdots, 0)$ and $\mathbf{y}$. Note that $g\left(\theta^{\prime}\right)$ is positive definite in $\mathbb{S}^{d}$, and $P_{n}^{\alpha}\left(\cos \theta^{\prime}\right)$ is also positive definite on $\mathbb{S}^{d}$, so is their product. By lemma (4.1.2), $D_{n}$ is nonnegative, so are all coefficients of (4.1.14). Thus, (4.1.14) may be rewritten as

$$
g(\vartheta) \sim \sum_{n=0}^{\infty} b_{n} P_{n}^{\alpha}(\cos \vartheta)
$$

where $b_{n}$ is the same as in (4.2.1).

On the other hand, we know this series is Abel-summable for all $\vartheta$, hence, in particular, for $\vartheta=0$. Thus

$$
\sum_{n=0}^{k} b_{n}\left|P_{n}^{\alpha}(\cos \vartheta)\right| \leq \sum_{n=0}^{k} b_{n} P_{n}^{(\alpha)}(1) \leq \lim _{r \rightarrow 1^{-}} \sum_{n=0}^{\infty} b_{n} r^{n} P_{n}^{(\alpha)}(1)=g(1) .
$$

This shows that the series (4.2.3) is absolutely and uniformly convergent for all $\vartheta$, hence convergent to its Abel-sum which is $g(\vartheta)$.

The other side of prove is by the property i in theorem 3.4.1. Note that $g(\vartheta)$ is continuous because the series (4.2.2) must be convergent uniformly. As $g(\vartheta)$ is the continuous limit of a sequence of positive definite functions, it is also positive definite.

\subsection{Recent Results}

In [15], it has been proved a compactly supported continuous function is positive definite on $\mathbb{S}^{d}$, if the function is positive definite in $\mathbb{R}^{d}$, where $d=1,3$.

In [28], Xu showed that the integral of Jacobi polynomials defined as

$$
F_{n}^{(\alpha, \beta), \delta}=\int_{0}^{a}(a-\vartheta)^{\delta} P_{n}^{\left(\alpha-\frac{1}{2}, \beta-\frac{1}{2}\right)}(\cos \vartheta)\left(\sin \frac{\vartheta}{2}\right)^{2 \alpha}\left(\cos \frac{\vartheta}{2}\right)^{2 \beta} d \vartheta
$$

is nonnegative. It can be used to prove the truncated power function

$$
g(x)=(a-\vartheta)_{+}^{\delta}= \begin{cases}(a-\vartheta)^{\delta}, & 0 \leq \vartheta \leq a, \\ 0, & \vartheta>a,\end{cases}
$$


is positive definite on $\mathbb{S}^{d-1}$ for $\delta>\frac{d}{2}+\frac{1}{2}$. 


\section{CHAPTER 5}

\section{ISOTROPIC POSITIVE DEFINITE FUNCTIONS ON SPHERES GENERATED FROM THOSE IN EUCLIDEAN SPACES}

\subsection{Main Results}

One of the main results in this thesis is the following theorem, which provides an approach for generating isotropic positive definite functions on the sphere from those on the Euclidean space.

Theorem 5.1.1. Suppose that $g(x)$ is a continuous function on $[0, \infty)$, and $g(x)=0, x \geq \pi$. For an odd integer $d$, if $g(\|\mathbf{x}\|)$ is a positive definite function in $\mathbb{R}^{d}$, then $g(\vartheta), \vartheta \in[0, \pi]$, is a positive definite function on $\mathbb{S}^{d}$.

Note: The variables $\mathbf{x}$ in 5.1 .1 can be any point in $\mathbb{R}^{d}$, but we should have $\left\|\mathbf{x}_{1}-\mathbf{x}_{2}\right\| \in$ $[0, \pi]$ in order to keep $g(\|\mathbf{x}\|)$ is positive definite in $\mathbb{R}^{d}$. For the function $g(x)=\cos x$, which is isotropic positive definite in $R$ and on $\mathbb{S}^{1}$, it is not satisfies the conditions of 5.1.1, since $g(\pi)=-1$

It is not clear whether a similar result holds for an even integer $d$. Nevertheless, the next corollary is a consequence of Theorem 5.1.1, since an isotropic positive definite function in $\mathbb{R}^{d}$ is also an isotropic positive definite function in $\mathbb{R}^{d-1}(d \geq 2)$, and $d-1$ is odd for an even $d$.

Corollary 1. Let $g(x)$ be as in Theorem 5.1.1. For an even integer d, if $g(\|\mathbf{x}\|)$ is a positive definite function in $\mathbb{R}^{d}$, then $g(\vartheta), \vartheta \in[0, \pi]$, is a positive definite function on $\mathbb{S}^{d-1}$.

Although the requirement $g(x)=0, x \geq \pi$, is crucial in Theorem 5.1.1, it is always possible to change the scale for a compactly supported function. This result is in the following corollary.

Corollary 2. Let $g(x)$ be a continuous function on $[0, \infty)$, and $g(x)=0, x \geq l$. For an odd integer $d$, if $g(\|\mathbf{x}\|)$ is a positive definite function in $\mathbb{R}^{d}$, then $g\left(\frac{l}{\pi} \vartheta\right), \vartheta \in[0, \pi]$, is a positive definite function on $\mathbb{S}^{d}$. 
Since $g(x)$ is continuous with compact support on $[0, \infty)$, the positive definiteness of $g(\|\mathbf{x}\|)$ in $\mathbb{R}^{d}$ implies that

$$
\int_{0}^{\pi} u^{\frac{d}{2}} J_{\frac{d}{2}-1}(x u) g(u) d u \geq 0, \quad x \geq 0
$$

Hence, Theorem 5.1.1 can be rephrased as the two inequality implication statement as follows, in term of the Bessel function and the ultraspherical polynomial.

Theorem 5.1.2. For a nonnegative integer $\alpha$ and a continuous function $g(x)$ on $[0, \pi]$ with $g(\pi)=0$, if

$$
\int_{0}^{\pi} u^{\alpha+\frac{1}{2}} J_{\alpha-\frac{1}{2}}(x u) g(u) d u \geq 0, \quad x \geq 0
$$

then

$$
b_{n}=\int_{0}^{\pi} g(\vartheta) P_{n}^{(\alpha)}(\cos \vartheta) \sin ^{2 \alpha} \vartheta d \vartheta \geq 0, \quad n \in \mathbb{N}_{0}
$$

and $\sum_{n=0}^{\infty} b_{n} P_{n}^{(\alpha)}(1)$ converges.

Moreover, if inequality (5.1.1) is strict for every $x \geq 0$, then so is inequality (5.1.2) for every $n \in \mathbb{N}_{0}$.

The proof of Theorems 5.1.1 and 5.1.2 occupies Section 4, for which we need some lemmas in Section 3 and Theorem 5.1.3 that builds a useful bridge between the Jacobi polynomial and the Bessel function, as an extension of Theorem 8.1.1 of [25].

The base for an isotropic positive definite function in $\mathbb{R}^{d}$ is the Bessel function, as is released by the integral representation (1.0.1), and the base for that on $\mathbb{S}^{d}$ is the ultraspherical polynomial, by (1.0.4). The latter is a special case of the Jacobi polynomial. To prove Theorem 5.1.1 or 5.1.2 we need a bridge to connect these two types of special functions. According to Theorem 8.1.1 of [25], the Bessel function is related to the Jacobi polynomial by

$$
\lim _{m \rightarrow \infty} m^{-\left(\alpha-\frac{1}{2}\right)} P_{m}^{\left(\alpha-\frac{1}{2}, \beta-\frac{1}{2}\right)}\left(\cos \frac{z}{m}\right)=\left(\frac{z}{2}\right)^{-\left(\alpha-\frac{1}{2}\right)} J_{\alpha-\frac{1}{2}}(z), \quad z \in \mathbb{C},
$$


where the limit holds uniformly in every bounded region of the complex z-plane $\mathbb{C}$. This relation is empolyed in [28] to deduce the positivity of the integrals of the Jacobi polynomial from that of the Bessel function. A generalized version of (5.1.3) is given in the next theorem, where $\gamma_{m}$ is assumed to be a rational number for ensuring that the degree, $m \gamma_{m}+\gamma_{0}$, of a Jacobi polynomial is a positive integer.

Theorem 5.1.3. For $\alpha>-\frac{1}{2}, \beta>-\frac{1}{2}$, and $\gamma_{0} \in \mathbb{N}_{0}$, if $\left\{\gamma_{m}, m \in \mathbb{N}\right\}$ is a sequence of bounded positive rational numbers such that $m \gamma_{m} \in \mathbb{N}$ and $\lim _{m \rightarrow \infty} \gamma_{m}=\gamma>0$, then

$$
\lim _{m \rightarrow \infty} m^{-\left(\alpha-\frac{1}{2}\right)} P_{m \gamma_{m}+\gamma_{0}}^{\left(\alpha-\frac{1}{2}, \beta-\frac{1}{2}\right)}\left(\cos \frac{z}{m}\right)=\left(\frac{z}{2}\right)^{-\left(\alpha-\frac{1}{2}\right)} J_{\alpha-\frac{1}{2}}(z \gamma), \quad z \in \mathbb{C}
$$

Proof. For $m \gamma_{m}+\gamma_{0} \in \mathbb{N}$, an explicit expression of $P_{m \gamma_{m}+\gamma_{0}}^{\left(\alpha-\frac{1}{2}, \beta-\frac{1}{2}\right)}(x)$ is given by formula (4.21.2) of [25], which reads,

$$
\begin{aligned}
& P_{m \gamma_{m}+\gamma_{0}}^{\left(\alpha-\frac{1}{2}, \beta-\frac{1}{2}\right)}(x) \\
& =\sum_{\nu=0}^{m \gamma_{m}+\gamma_{0}} \frac{1}{\nu ! \Gamma\left(\nu+\alpha+\frac{1}{2}\right)} \frac{\Gamma\left(m \gamma_{m}+\gamma_{0}+\alpha+\beta+\nu\right) \Gamma\left(m \gamma_{m}+\gamma_{0}+\alpha+\frac{1}{2}\right)}{\Gamma\left(m \gamma_{m}+\gamma_{0}+\alpha+\beta\right) \Gamma\left(m \gamma_{m}+\gamma_{0}-\nu+1\right)}\left(\frac{x-1}{2}\right)^{\nu} .
\end{aligned}
$$

after substituting $x$ by $\cos \frac{z}{m}$,

$$
\begin{aligned}
& P_{m \gamma_{m}+\gamma_{0}}^{\left(\alpha-\frac{1}{2}, \beta-\frac{1}{2}\right)}\left(\cos \frac{z}{m}\right) \\
= & \sum_{\nu=0}^{m \gamma_{m}+\gamma_{0}} \frac{1}{\nu ! \Gamma\left(\nu+\alpha+\frac{1}{2}\right)} \frac{\Gamma\left(m \gamma_{m}+\gamma_{0}+\alpha+\beta+\nu\right) \Gamma\left(m \gamma_{m}+\gamma_{0}+\alpha+\frac{1}{2}\right)}{\Gamma\left(m \gamma_{m}+\gamma_{0}+\alpha+\beta\right) \Gamma\left(m \gamma_{m}+\gamma_{0}-\nu+1\right)}\left(\frac{\cos \frac{z}{m}-1}{2}\right)^{\nu} \\
= & \sum_{\nu=0}^{m \gamma_{m}+\gamma_{0}} \frac{1}{\nu ! \Gamma\left(\nu+\alpha+\frac{1}{2}\right)} \frac{\Gamma\left(m \gamma_{m}+\gamma_{0}+\alpha+\beta+\nu\right) \Gamma\left(m \gamma_{m}+\gamma_{0}+\alpha+\frac{1}{2}\right)}{\Gamma\left(m \gamma_{m}+\gamma_{0}+\alpha+\beta\right) \Gamma\left(m \gamma_{m}+\gamma_{0}-\nu+1\right)}\left(-\sin ^{2} \frac{z}{2 m}\right)^{\nu}
\end{aligned}
$$

Keeping $\nu$ and $z$ fixed, and letting $m \rightarrow \infty$, by the asymptotic formula $(2.2 .2), \frac{\Gamma(n+k)}{\Gamma(n)} \sim n^{k}$ and $\sin \frac{z}{2 m} \sim \frac{z}{2 m}$, we obtain the following asymptotic expression for the $(\nu+1)$ st term in the 
above series,

$$
\begin{aligned}
& \frac{1}{\nu ! \Gamma\left(\nu+\alpha+\frac{1}{2}\right)} \frac{\Gamma\left(m \gamma_{m}+\gamma_{0}+\alpha+\beta+\nu\right) \Gamma\left(m \gamma_{m}+\gamma_{0}+\alpha+\frac{1}{2}\right)}{\Gamma\left(m \gamma_{m}+\gamma_{0}+\alpha+\beta\right) \Gamma\left(m \gamma_{m}+\gamma_{0}-\nu+1\right)}\left(-\sin ^{2} \frac{z}{2 m}\right)^{\nu} \\
\sim & \frac{1}{\nu ! \Gamma\left(\nu+\alpha+\frac{1}{2}\right)} \frac{\Gamma\left(m \gamma+\gamma_{0}+\alpha+\beta+\nu\right) \Gamma\left(m \gamma+\gamma_{0}+\alpha+\frac{1}{2}\right)}{\Gamma\left(m \gamma+\gamma_{0}+\alpha+\beta\right) \Gamma\left(m \gamma+\gamma_{0}-\nu+1\right)}\left(\frac{z}{2 m}\right)^{2 \nu} \\
\sim & \frac{(m \gamma)^{\nu}(m \gamma)^{\nu+\alpha-\frac{1}{2}}}{\nu ! \Gamma\left(\nu+\alpha+\frac{1}{2}\right)}\left(\frac{z}{2 m}\right)^{2 \nu} \\
\sim & \frac{(-1)^{\nu}(m \gamma)^{\alpha-\frac{1}{2}}}{\nu ! \Gamma\left(\nu+\alpha+\frac{1}{2}\right)}\left(\frac{z \gamma}{2}\right)^{2 \nu},
\end{aligned}
$$

and

$$
\begin{aligned}
& \lim _{m \rightarrow \infty} m^{-\left(\alpha-\frac{1}{2}\right)} P_{m \gamma_{m}+\gamma_{0}}^{\left(\alpha-\frac{1}{2}, \beta-\frac{1}{2}\right)}\left(\cos \frac{z}{m}\right) \\
= & \lim _{m \rightarrow \infty} \sum_{\nu=0}^{m \gamma_{m}+\gamma_{0}} \frac{m^{-\left(\alpha-\frac{1}{2}\right)}}{\nu ! \Gamma\left(\nu+\alpha+\frac{1}{2}\right)} \frac{\Gamma\left(m \gamma_{m}+\gamma_{0}+\alpha+\beta+\nu\right) \Gamma\left(m \gamma_{m}+\gamma_{0}+\alpha+\frac{1}{2}\right)}{\Gamma\left(m \gamma_{m}+\gamma_{0}+\alpha+\beta\right) \Gamma\left(m \gamma_{m}+\gamma_{0}-\nu+1\right)}\left(-\sin ^{2} \frac{z}{2 m}\right)^{\nu} \\
= & \sum_{\nu=0}^{\infty} \frac{(-1)^{\nu} \gamma^{\alpha-\frac{1}{2}}}{\nu ! \Gamma\left(\nu+\alpha+\frac{1}{2}\right)}\left(\frac{z \gamma}{2}\right)^{2 \nu} \\
= & \left(\frac{z}{2}\right)^{-\left(\alpha-\frac{1}{2}\right)} J_{\alpha-\frac{1}{2}}(z \gamma),
\end{aligned}
$$

where passing to the limit under the summation sign is valid, due to the existence of a dominant for the total sum which is readily derived. Indeed if $m$ is large enough, then we have

$$
\begin{aligned}
& \frac{m^{-\left(\alpha-\frac{1}{2}\right)}}{\nu !\left(m \gamma+\gamma_{0}-\nu\right) !} \frac{\Gamma\left(m \gamma+\gamma_{0}+\alpha+\beta+\nu\right) \Gamma\left(m \gamma+\gamma_{0}+\alpha+\frac{1}{2}\right)}{\Gamma\left(m \gamma+\gamma_{0}+\alpha+\beta\right) \Gamma\left(\nu+\alpha+\frac{1}{2}\right)}\left(\frac{z}{2 m}\right)^{2 \nu} \\
\leq & \frac{\left(2 m \gamma+2 \gamma_{0}+\alpha+\beta\right)^{\nu} \Gamma\left(m \gamma+\gamma_{0}+\alpha+\frac{1}{2}\right) z^{2 \nu}}{\nu !\left(m \gamma+\gamma_{0}-\nu\right) ! m^{\alpha-\frac{1}{2}} \Gamma\left(\nu+\alpha+\frac{1}{2}\right)(2 m)^{2 \nu}} \\
\leq & \frac{\left(m \gamma+\gamma_{0}\right)^{\nu}\left(2 m \gamma+2 \gamma_{0}+\alpha+\beta\right)^{\nu} \Gamma\left(m \gamma+\gamma_{0}+\alpha+\frac{1}{2}\right) z^{2 \nu}}{\nu !\left(m \gamma+\gamma_{0}\right) ! m^{\alpha-\frac{1}{2}} \Gamma\left(\nu+\alpha+\frac{1}{2}\right)(2 m)^{2 \nu}} \\
= & O(1) .
\end{aligned}
$$




\subsection{Some Lemmas and Their Proofs}

In order to prove Theorems 5.1.1 and 5.1.2 in the next section, we need some lemmas involving the Jacobi polynomial and recurrent formulas related to $b_{n}$ in (5.1.2), besides Theorem 5.1.3.

For a nonnegative integer $\beta$, Lemma 5.2.1 expresses the $n+\beta+1$ degree polynomial $(1+x)^{\beta+1} P_{n}^{\left(\alpha-\frac{1}{2}, \beta+\frac{1}{2}\right)}(x)$ as a nonnegative combination of polynomials $P_{n+j}^{\left(\alpha-\frac{1}{2},-\frac{1}{2}\right)}(x), j=$ $0,1, \ldots, \beta+1$.

Lemma 5.2.1. For $\alpha>-\frac{1}{2}$ and $\beta \in \mathbb{N}_{0}$,

$$
(1+x)^{\beta+1} P_{n}^{\left(\alpha-\frac{1}{2}, \beta+\frac{1}{2}\right)}(x)=\sum_{j=0}^{\beta+1} a_{j, n}^{\alpha, \beta} P_{n+j}^{\left(\alpha-\frac{1}{2},-\frac{1}{2}\right)}(x), \quad x \in \mathbb{R}, n \in \mathbb{N}_{0},
$$

where

$$
a_{j, n}^{\alpha, \beta}=\frac{2^{\beta+1}(\alpha+2 n+2 j)(n+j) ! \Gamma(\alpha+2 n+j) \Gamma\left(\beta+n+\frac{3}{2}\right) \Gamma(\beta+2)}{n ! j ! \Gamma(\alpha+\beta+2 n+j+2) \Gamma(\beta-j+2) \Gamma\left(n+j+\frac{1}{2}\right)} .
$$

Proof. We will give two proofs of the Lemma. The first one is by mathematical induction, in the second one we use some identities from the references.

Proof 1. The proof of (5.2.1) is by induction on $\beta$.

For $\beta=0,(5.2 .1)$ appears as identity (22.7.16) of [1],

$$
\begin{gathered}
(2 n+\alpha+\beta+1)(1+x) P_{n}^{\left(\alpha-\frac{1}{2}, \beta+\frac{1}{2}\right)}(x) \\
=(2 n+2 \beta+1) P_{n}^{\left(\alpha-\frac{1}{2}, \beta-\frac{1}{2}\right)}(x)+2(n+1) P_{n+1}^{\left(\alpha-\frac{1}{2}, \beta-\frac{1}{2}\right)}(x) .
\end{gathered}
$$

or,

$$
\begin{aligned}
& (1+x) P_{n}^{\left(\alpha-\frac{1}{2}, \beta+\frac{1}{2}\right)}(x) \\
= & \frac{2 n+2 \beta+1}{2 n+\alpha+\beta+1} P_{n}^{\left(\alpha-\frac{1}{2}, \beta-\frac{1}{2}\right)}(x)+\frac{2(n+1)}{2 n+\alpha+\beta+1} P_{n+1}^{\left(\alpha-\frac{1}{2}, \beta-\frac{1}{2}\right)}(x) .
\end{aligned}
$$

Taking $\beta=0$, we have

$$
\begin{aligned}
& (1+x) P_{n}^{\left(\alpha-\frac{1}{2}, \frac{1}{2}\right)}(x) \\
= & \frac{2 n+1}{2 n+\alpha+1} P_{n}^{\left(\alpha-\frac{1}{2},-\frac{1}{2}\right)}(x)+\frac{2(n+1)}{2 n+\alpha+1} P_{n+1}^{\left(\alpha-\frac{1}{2},-\frac{1}{2}\right)}(x),
\end{aligned}
$$


where

$$
a_{0, n}^{\alpha, 0}=\frac{2 n+1}{2 n+\alpha+1}
$$

and

$$
a_{1, n}^{\alpha, 0}=\frac{2(n+1)}{2 n+\alpha+1} .
$$

Assume that (5.2.1) is true for $\beta=\lambda-1$, i.e,

$$
(1+x)^{\lambda} P_{n}^{\left(\alpha-\frac{1}{2}, \lambda-\frac{1}{2}\right)}(x)=\sum_{j=0}^{\lambda} a_{j, n}^{\alpha, \lambda-1} P_{n+j}^{\left(\alpha-\frac{1}{2},-\frac{1}{2}\right)}(x),
$$

substituting $n$ by $n+1$,

$$
(1+x)^{\lambda} P_{n+1}^{\left(\alpha-\frac{1}{2}, \lambda-\frac{1}{2}\right)}(x)=\sum_{j=0}^{\lambda} a_{j, n+1}^{\alpha, \lambda-1} P_{n+1+j}^{\left(\alpha-\frac{1}{2},-\frac{1}{2}\right)}(x) .
$$

Then for $\beta=\lambda$, by the identity (5.2.3)

$$
\begin{aligned}
& (1+x)^{\lambda+1} P_{n}^{\left(\alpha-\frac{1}{2}, \lambda+\frac{1}{2}\right)}(x) \\
= & (1+x)(1+x)^{\lambda} P_{n}^{\left(\alpha-\frac{1}{2}, \lambda+\frac{1}{2}\right)}(x) \\
= & \frac{2 n+2 \lambda+1}{2 n+\alpha+\lambda+1}(1+x)^{\lambda} P_{n}^{\left(\alpha-\frac{1}{2}, \lambda-\frac{1}{2}\right)}(x)+\frac{2(n+1)}{2 n+\alpha+\lambda+1}(1+x)^{\lambda} P_{n+1}^{\left(\alpha-\frac{1}{2}, \lambda-\frac{1}{2}\right)}(x) \\
= & \frac{2 n+2 \lambda+1}{2 n+\alpha+\lambda+1} \sum_{j=0}^{\lambda} a_{j, n}^{\alpha, \lambda-1} P_{n+j}^{\left(\alpha-\frac{1}{2},-\frac{1}{2}\right)}(x)+\frac{2(n+1)}{2 n+\alpha+\lambda+1} \sum_{j=0}^{\lambda} a_{j, n+1}^{\alpha, \lambda-1} P_{n+1+j}^{\left(\alpha-\frac{1}{2},-\frac{1}{2}\right)}(x) \\
= & \frac{2 n+2 \lambda+1}{2 n+\alpha+\lambda+1} a_{0, n}^{\alpha, \lambda-1} P_{n}^{\left(\alpha-\frac{1}{2},-\frac{1}{2}\right)}(x)+\frac{2(n+1)}{2 n+\alpha+\lambda+1} a_{\lambda, n+1}^{\alpha, \lambda-1} P_{n+1+\lambda}^{\left(\alpha-\frac{1}{2},-\frac{1}{2}\right)}(x) \\
+ & \frac{2 n+2 \lambda+1}{2 n+\alpha+\lambda+1} \sum_{j=1}^{\lambda} a_{j, n}^{\alpha, \lambda-1} P_{n+j}^{\left(\alpha-\frac{1}{2},-\frac{1}{2}\right)}(x)+\frac{2(n+1)}{2 n+\alpha+\lambda+1} \sum_{j=0}^{\lambda-1} a_{j, n+1}^{\alpha, \lambda-1} P_{n+1+j}^{\left(\alpha-\frac{1}{2},-\frac{1}{2}\right)}(x) \\
= & \frac{2 n+2 \lambda+1}{2 n+\alpha+\lambda+1} a_{0, n}^{\alpha, \lambda-1} P_{n}^{\left(\alpha-\frac{1}{2},-\frac{1}{2}\right)}(x)+\frac{2(n+1)}{2 n+\alpha+\lambda+1} a_{\lambda, n+1}^{\alpha, \lambda-1} P_{n+1+\lambda}^{\left(\alpha-\frac{1}{2},-\frac{1}{2}\right)}(x) \\
+ & \frac{2 n+2 \lambda+1}{2 n+\alpha+\lambda+1} \sum_{j=1}^{\lambda} a_{j, n}^{\alpha, \lambda-1} P_{n+j}^{\left(\alpha-\frac{1}{2},-\frac{1}{2}\right)}(x)+\frac{2(n+1)}{2 n+\alpha+\lambda+1} \sum_{j=1}^{\lambda} a_{j-1, n+1}^{\alpha, \lambda-1} P_{n+j}^{\left(\alpha-\frac{1}{2},-\frac{1}{2}\right)}(x) \\
= & \frac{2 n+2 \lambda+1}{2 n+\alpha+\lambda+1} a_{0, n}^{\alpha, \lambda-1} P_{n}^{\left(\alpha-\frac{1}{2},-\frac{1}{2}\right)}(x)+\frac{2(n+1)}{2 n+\alpha+\lambda+1} a_{\lambda, n+1}^{\alpha, \lambda-1} P_{n+1+\lambda}^{\left(\alpha-\frac{1}{2},-\frac{1}{2}\right)}(x) \\
+ & \sum_{j=1}^{\lambda}\left(\frac{2 n+2 \lambda+1}{2 n+\alpha+\lambda+1} a_{j, n}^{\alpha, \lambda-1}+\frac{2(n+1)}{2 n+\alpha+\lambda+1} a_{j-1, n+1}^{\alpha, \lambda-1}\right) P_{n+j}^{\left(\alpha-\frac{1}{2},-\frac{1}{2}\right)}(x) \\
= & \sum_{j=0}^{\lambda+1} a_{j, n}^{\alpha, \lambda} P_{n+j}^{\left(\alpha-\frac{1}{2}, \lambda-\frac{1}{2}\right)}(x) \\
&
\end{aligned}
$$


where

$$
\begin{gathered}
a_{0, n}^{\alpha, \lambda}=\frac{2 n+2 \lambda+1}{2 n+\alpha+\lambda+1} a_{0, n}^{\alpha, \lambda-1}, \\
a_{\lambda+1, n}^{\alpha, \lambda}=\frac{2(n+1)}{2 n+\alpha+\lambda+1} a_{\lambda, n+1}^{\alpha, \lambda-1},
\end{gathered}
$$

and

$$
a_{j, n}^{\alpha, \lambda}=\frac{2 n+2 \lambda+1}{2 n+\alpha+\lambda+1} a_{j, n}^{\alpha, \lambda-1}+\frac{2(n+1)}{2 n+\alpha+\lambda+1} a_{j-1, n+1}^{\alpha, \lambda-1}
$$

for $j=1,2, \cdots, \lambda$.

Proof 2. Successively using this identity (5.2.3), the $n+\beta+1$ degree polynomial $(1+$ $x)^{\beta} P_{n}^{\left(\alpha-\frac{1}{2}, \beta+\frac{1}{2}\right)}(x)$ can be written as a linear combination of polynomials $P_{n+j}^{\left(\alpha-\frac{1}{2},-\frac{1}{2}\right)}(x), j=$ $0,1, \ldots, \beta+1$. To verify (5.2.2) for $a_{j, n}^{\alpha, \beta}$ in (5.2.1), we multiply both sides of (5.2.1) by $(1-x)^{\alpha-\frac{1}{2}}(1+x)^{-\frac{1}{2}} P_{n+j}^{\left(\alpha-\frac{1}{2},-\frac{1}{2}\right)}(x)$ and then integrate from -1 and 1,

$$
\begin{aligned}
& \frac{2^{\alpha} \Gamma\left(\alpha+n+j+\frac{1}{2}\right) \Gamma\left(n+j+\frac{1}{2}\right)}{(n+j) !(\alpha+2 n+2 j) \Gamma(\alpha+n+j)} a_{j, n}^{\alpha, \beta} \\
= & \sum_{k=0}^{\beta+1} a_{k, n}^{\alpha, \beta} \int_{-1}^{1}(1-x)^{\alpha-\frac{1}{2}}(1+x)^{-\frac{1}{2}} P_{n+j}^{\left(\alpha-\frac{1}{2},-\frac{1}{2}\right)}(x) P_{n+k}^{\left(\alpha-\frac{1}{2},-\frac{1}{2}\right)}(x) d x \\
= & \int_{-1}^{1}(1-x)^{\alpha-\frac{1}{2}}(1+x)^{\beta+\frac{1}{2}} P_{n+j}^{\left(\alpha-\frac{1}{2},-\frac{1}{2}\right)}(x) P_{n}^{\left(\alpha-\frac{1}{2}, \beta+\frac{1}{2}\right)}(x) d x \\
= & \frac{2^{\alpha+\beta+1} \Gamma\left(\alpha+n+j+\frac{1}{2}\right) \Gamma(\alpha+2 n+j) \Gamma\left(\beta+n+\frac{3}{2}\right) \Gamma(\beta+2)}{n ! j ! \Gamma(\alpha+n+j) \Gamma(\alpha+\beta+2 n+j+2) \Gamma(\beta-j+2)},
\end{aligned}
$$

where the first equality is obtained from identity (7.391.1) of [13], and the last equality from identity (7.391.9) of [13].

Suppose that $g(\vartheta)$ is a continuous function on $[0, \infty)$ and $g(\vartheta)=0, \vartheta \geq \pi$. For $\alpha, \beta>-\frac{1}{2}$, define

$$
b_{n, k}^{(\alpha, \beta)}=\int_{0}^{\pi} g(\vartheta) P_{n}^{\left(\alpha-\frac{1}{2}, \beta-\frac{1}{2}\right)}\left(\cos \frac{\vartheta}{2^{k}}\right)\left(\sin \frac{\vartheta}{2^{k+1}}\right)^{2 \alpha}\left(\cos \frac{\vartheta}{2^{k+1}}\right)^{2 \beta} d \vartheta, \quad n \in \mathbb{N}_{0}, k \in \mathbb{N}_{0},
$$


and, for simplicity, write $b_{n}^{(\alpha, \beta)}$ for $b_{n, 0}^{(\alpha, \beta)}$ when $k=0$. Thus,

$$
b_{n}^{(\alpha, \beta)}=b_{n, 0}^{(\alpha, \beta)}=\int_{0}^{\pi} g(\vartheta) P_{n}^{\left(\alpha-\frac{1}{2}, \beta-\frac{1}{2}\right)}(\cos \vartheta)\left(\sin \frac{\vartheta}{2}\right)^{2 \alpha}\left(\cos \frac{\vartheta}{2}\right)^{2 \beta} d \vartheta
$$

and, for $b_{n}$ in (5.1.2),

$$
b_{n}=\frac{2^{2 \alpha} \Gamma\left(\alpha+\frac{1}{2}\right) \Gamma(n+2 \alpha)}{\Gamma(2 \alpha) \Gamma\left(n+\alpha+\frac{1}{2}\right)} b_{n}^{(\alpha, \alpha)}, \quad n \in \mathbb{N}_{0},
$$

which is a direct result of (1.0.6).

Lemma 5.2.2. For $\alpha, \beta>-\frac{1}{2}$,

$$
b_{n}^{(\alpha, \beta+1)}=\frac{2 n+2 \beta+1}{2(2 n+\alpha+\beta+1)} b_{n}^{(\alpha, \beta)}+\frac{n+1}{2 n+\alpha+\beta+1} b_{n+1}^{(\alpha, \beta)}, \quad n \in \mathbb{N}_{0} .
$$

If $\alpha>-\frac{1}{2}$ and $\beta \in \mathbb{N}_{0}$, then

$$
b_{n, k}^{(\alpha, \beta+1)}=2^{-(\beta+1)} \sum_{j=0}^{\beta+1} a_{j, n}^{\alpha, \beta} b_{n+j, k}^{(\alpha, 0)}, \quad n \in \mathbb{N}_{0}, k \in \mathbb{N}_{0}
$$

and, in particular, for $\alpha \in \mathbb{N}$,

$$
b_{n, k}^{(\alpha, \alpha)}=2^{-\alpha} \sum_{j=0}^{\alpha} a_{j, n}^{\alpha, \alpha-1} b_{n+j, k}^{(\alpha, 0)}, \quad n \in \mathbb{N}_{0}, k \in \mathbb{N}_{0}
$$

and

$$
b_{n}^{(\alpha, \alpha)}=2^{-\alpha} \sum_{j=0}^{\alpha} a_{j, n}^{\alpha, \alpha-1} b_{n+j}^{(\alpha, 0)}
$$

where $a_{j, n}^{\alpha, \beta}$ 's are positive constants defined in (5.2.2).

Proof. Substituting $x$ by $\cos \vartheta$ in (5.2.3) yields

$$
\begin{gathered}
(2 n+\alpha+\beta+1)(1+\cos \vartheta) P_{n}^{\left(\alpha-\frac{1}{2}, \beta+\frac{1}{2}\right)}(\cos \vartheta) \\
=(2 n+2 \beta+1) P_{n}^{\left(\alpha-\frac{1}{2}, \beta-\frac{1}{2}\right)}(\cos \vartheta)+2(n+1) P_{n+1}^{\left(\alpha-\frac{1}{2}, \beta-\frac{1}{2}\right)}(\cos \vartheta)
\end{gathered}
$$

or

$$
2(2 n+\alpha+\beta+1) \cos ^{2}\left(\frac{\vartheta}{2}\right) P_{n}^{\left(\alpha-\frac{1}{2}, \beta+\frac{1}{2}\right)}(\cos \vartheta)
$$




$$
=(2 n+2 \beta+1) P_{n}^{\left(\alpha-\frac{1}{2}, \beta-\frac{1}{2}\right)}(\cos \vartheta)+2(n+1) P_{n+1}^{\left(\alpha-\frac{1}{2}, \beta-\frac{1}{2}\right)}(\cos \vartheta)
$$

Multiplying both sides of the last equation by $g(\vartheta)\left(\sin \frac{\vartheta}{2}\right)^{2 \alpha}\left(\cos \frac{\vartheta}{2}\right)^{2 \beta}$ and integrating from 0 to $\pi$, we obtain $(5.2 .4)$.

To derive (5.2.5), we substitute $x$ by $\cos \left(\frac{\vartheta}{2^{k}}\right)$ in $(5.2 .1)$,

$$
\begin{aligned}
& \sum_{j=0}^{\beta+1} a_{j, n}^{\alpha, \beta} P_{n+j}^{\left(\alpha-\frac{1}{2},-\frac{1}{2}\right)}\left(\cos \frac{\vartheta}{2^{k}}\right) \\
= & \left(1+\cos \frac{\vartheta}{2^{k}}\right)^{\beta+1} P_{n}^{\left(\alpha-\frac{1}{2}, \beta+\frac{1}{2}\right)}\left(\cos \frac{\vartheta}{2^{k}}\right) \\
= & 2^{\beta+1}\left(\cos \frac{\vartheta}{2^{k+1}}\right)^{2(\beta+1)} P_{n}^{\left(\alpha-\frac{1}{2}, \beta+\frac{1}{2}\right)}\left(\cos \frac{\vartheta}{2^{k}}\right),
\end{aligned}
$$

and obtain

$$
\begin{aligned}
b_{n, k}^{(\alpha, \beta+1)} & =\int_{0}^{\pi} g(\vartheta)\left(\cos \frac{\vartheta}{2^{k+1}}\right)^{2(\beta+1)} P_{n}^{\left(\alpha-\frac{1}{2}, \beta+\frac{1}{2}\right)}\left(\cos \frac{\vartheta}{2^{k}}\right)\left(\sin \frac{\vartheta}{2^{k+1}}\right)^{2 \alpha} d \vartheta \\
& =2^{-(\beta+1)} \sum_{j=0}^{\beta+1} a_{j, n}^{\alpha, \beta} \int_{0}^{\pi} g(\vartheta) P_{n+j}^{\left(\alpha-\frac{1}{2},-\frac{1}{2}\right)}\left(\cos \frac{\vartheta}{2^{k}}\right)\left(\sin \frac{\vartheta}{2^{k+1}}\right)^{2 \alpha} d \vartheta \\
& =2^{-(\beta+1)} \sum_{j=0}^{\beta+1} a_{j, n}^{\alpha, \beta} b_{n+j, k}^{(\alpha, 0)} .
\end{aligned}
$$

Lemma 5.2.3. For $\alpha>-\frac{1}{2}$ and $n, k \in \mathbb{N}_{0}$,

$$
b_{2 n, k+1}^{(\alpha, \alpha)}=\frac{\Gamma\left(2 n+\alpha+\frac{1}{2}\right) \Gamma(n+1)}{2^{2 \alpha} \Gamma\left(n+\alpha+\frac{1}{2}\right) \Gamma(2 n+1)} b_{n, k}^{(\alpha, 0)},
$$

and, in particular,

$$
b_{2 n, 1}^{(\alpha, \alpha)}=\frac{\Gamma\left(2 n+\alpha+\frac{1}{2}\right) \Gamma(n+1)}{2^{2 \alpha} \Gamma\left(n+\alpha+\frac{1}{2}\right) \Gamma(2 n+1)} b_{n}^{(\alpha, 0)} .
$$

Proof. By identity (4.1.5) of [25],

$$
P_{2 n}^{\left(\alpha-\frac{1}{2}, \alpha-\frac{1}{2}\right)}(x)=\frac{\Gamma\left(2 n+\alpha+\frac{1}{2}\right) \Gamma(n+1)}{\Gamma\left(n+\alpha+\frac{1}{2}\right) \Gamma(2 n+1)} P_{n}^{\left(\alpha-\frac{1}{2},-\frac{1}{2}\right)}\left(2 x^{2}-1\right)
$$


which reads, after substituting $x$ by $\cos \frac{\vartheta}{2^{k+1}}$,

$$
P_{2 n}^{\left(\alpha-\frac{1}{2}, \alpha-\frac{1}{2}\right)}\left(\cos \frac{\vartheta}{2^{k+1}}\right)=\frac{\Gamma\left(2 n+\alpha+\frac{1}{2}\right) \Gamma(n+1)}{\Gamma\left(n+\alpha+\frac{1}{2}\right) \Gamma(2 n+1)} P_{n}^{\left(\alpha-\frac{1}{2},-\frac{1}{2}\right)}\left(\cos \frac{\vartheta}{2^{k}}\right) .
$$

From the last equality we obtain

$$
\begin{aligned}
b_{2 n, k+1}^{(\alpha, \alpha)} & =\int_{0}^{\pi} g(\vartheta) P_{2 n}^{\left(\alpha-\frac{1}{2}, \alpha-\frac{1}{2}\right)}\left(\cos \frac{\vartheta}{2^{k+1}}\right)\left(\sin \frac{\vartheta}{2^{k+2}} \cos \frac{\vartheta}{2^{k+2}}\right)^{2 \alpha} d \vartheta \\
& =2^{-2 \alpha} \int_{0}^{\pi} g(\vartheta) P_{2 n}^{\left(\alpha-\frac{1}{2}, \alpha-\frac{1}{2}\right)}\left(\cos \frac{\vartheta}{2^{k+1}}\right)\left(\sin \frac{\vartheta}{2^{k+1}}\right)^{2 \alpha} d \vartheta \\
& =\frac{\Gamma\left(2 n+\alpha+\frac{1}{2}\right) \Gamma(n+1)}{2^{2 \alpha} \Gamma\left(n+\alpha+\frac{1}{2}\right) \Gamma(2 n+1)} \int_{0}^{\pi} g(\vartheta) P_{n}^{\left(\alpha-\frac{1}{2},-\frac{1}{2}\right)}\left(\cos \frac{\vartheta}{2^{k}}\right)\left(\sin \frac{\vartheta}{2^{k+1}}\right)^{2 \alpha} d \vartheta \\
& =\frac{\Gamma\left(2 n+\alpha+\frac{1}{2}\right) \Gamma(n+1)}{2^{2 \alpha} \Gamma\left(n+\alpha+\frac{1}{2}\right) \Gamma(2 n+1)} b_{n, k}^{(\alpha, 0)} .
\end{aligned}
$$

Lemma 5.2.4. For $\alpha \in \mathbb{N}, k \in \mathbb{N}$, and $n \in \mathbb{N}_{0}$,

$$
\begin{aligned}
b_{n}^{(\alpha, \alpha)}= & 2^{(k-1) \alpha} \sum_{j_{0}=0}^{\alpha} \ldots \sum_{j_{k}=0}^{\alpha} a_{j_{0}, n}^{\alpha, \alpha-1} \cdots a_{j_{k}, 2^{k}\left(n+j_{0}\right)+2^{k-1} j_{1}+\ldots+2 j_{k-1}}^{\alpha, \alpha-1} \\
& \times c\left(j_{0}, \ldots, j_{k}, n\right) b_{2^{k}\left(n+j_{0}\right)+2^{k-1} j_{1}+\ldots+j_{k}, k}^{(\alpha, 0)}
\end{aligned}
$$

where

$$
\begin{aligned}
c\left(j_{0}, j_{1}, \ldots, j_{k}, n\right) \\
=\prod_{l=0}^{k-1} \frac{\Gamma\left(2^{l}\left(n+j_{0}\right)+2^{l-1} j_{1}+\ldots+j_{l}+\alpha+\frac{1}{2}\right) \Gamma\left(2^{l+1}\left(n+j_{0}\right)+2^{l} j_{1}+\ldots+2 j_{l}+1\right)}{\Gamma\left(2^{l+1}\left(n+j_{0}\right)+2^{l} j_{1}+\ldots+2 j_{l}+\alpha+\frac{1}{2}\right) \Gamma\left(2^{l}\left(n+j_{0}\right)+2^{l-1} j_{1}+\ldots+j_{l}+1\right)}
\end{aligned}
$$

is a nonnegative constant and $a_{j_{k}, 2^{k}\left(n+j_{0}\right)+2^{k-1} j_{1}+\ldots+2 j_{k-1}}$ is defined as in (5.2.2) which is also nonegative.

Proof. The proof of (5.2.10) is by induction on $k$. For $k=1,(5.2 .10)$ follows from identities (5.2.7), (5.2.9) and (5.2.6)

$$
\begin{aligned}
& b_{n}^{(\alpha, \alpha)} \\
= & 2^{-\alpha} \sum_{j_{0}=0}^{\alpha} a_{j_{0}, n}^{\alpha, \alpha-1} b_{n+j_{0}}^{(\alpha, 0)} \\
= & 2^{-\alpha} \sum_{j_{0}=0}^{\alpha} a_{j_{0}, n}^{\alpha, \alpha-1} \frac{2^{2 \alpha} \Gamma\left(n+j_{0}+\alpha+\frac{1}{2}\right) \Gamma\left(2\left(n+j_{0}\right)+1\right)}{\Gamma\left(2\left(n+j_{0}\right)+\alpha+\frac{1}{2}\right) \Gamma\left(n+j_{0}+1\right)} b_{2\left(n+j_{0}\right), 1}^{(\alpha, \alpha)}
\end{aligned}
$$




$$
\begin{aligned}
& =2^{-\alpha} \sum_{j_{0}=0}^{\alpha} a_{j_{0}, n}^{\alpha, \alpha-1} \frac{2^{2 \alpha} \Gamma\left(n+j_{0}+\alpha+\frac{1}{2}\right) \Gamma\left(2\left(n+j_{0}\right)+1\right)}{\Gamma\left(2\left(n+j_{0}\right)+\alpha+\frac{1}{2}\right) \Gamma\left(n+j_{0}+1\right)} 2^{-\alpha} \sum_{j_{1}=0}^{\alpha} a_{j_{1}, 2\left(n+j_{0}\right)}^{\alpha, \alpha-1} b_{2\left(n+j_{0}\right)+j_{1}, 1}^{(\alpha, 0)} \\
& =\sum_{j_{0}=0}^{\alpha} a_{j_{0}, n}^{\alpha, \alpha-1} \sum_{j_{1}=0}^{\alpha} a_{j_{1}, 2\left(n+j_{0}\right)}^{\alpha, \alpha-1} \frac{\Gamma\left(n+j_{0}+\alpha+\frac{1}{2}\right) \Gamma\left(2\left(n+j_{0}\right)+1\right)}{\Gamma\left(2\left(n+j_{0}\right)+\alpha+\frac{1}{2}\right) \Gamma\left(n+j_{0}+1\right)} b_{2\left(n+j_{0}\right)+j_{1}, 1}^{(\alpha, 0)} .
\end{aligned}
$$

Suppose that (5.2.10) is true for $k=i$, i.e.,

$$
\begin{aligned}
b_{n}^{(\alpha, \alpha)}=2^{(i-1) \alpha} & \sum_{j_{0}=0}^{\alpha} \ldots \sum_{j_{i}=0}^{\alpha} a_{j_{0}, n}^{\alpha, \alpha-1} \cdots a_{j_{i}, 2^{i}\left(n+j_{0}\right)+2^{i-1} j_{1}+\ldots+2 j_{i-1}}^{\alpha, \alpha-1} \\
& \times c\left(j_{0}, \ldots, j_{i}, n\right) b_{2^{i}\left(n+j_{0}\right)+2^{i-1} j_{1}+\ldots+j_{i}, i}^{(\alpha, 0)}
\end{aligned}
$$

then for $k=i+1$, by (5.2.8) and (5.2.6)

$$
\begin{aligned}
& b_{n}^{(\alpha, \alpha)} \\
& =2^{(i-1) \alpha} \sum_{j_{0}=0}^{\alpha} \cdots \sum_{j_{i}=0}^{\alpha} a_{j_{0}, n}^{\alpha, \alpha-1} \cdots a_{j_{i}, 2^{i}\left(n+j_{0}\right)+2^{i-1} j_{1}+\ldots+2 j_{i-1}}^{\alpha, \alpha-1} \\
& \times c\left(j_{0}, \ldots, j_{i}, n\right) b_{2^{i}\left(n+j_{0}\right)+2^{i-1} j_{1}+\ldots+j_{i}, i}^{(\alpha, 0)} \\
& =2^{(i-1) \alpha} \sum_{j_{0}=0}^{\alpha} \ldots \sum_{j_{i}=0}^{\alpha} a_{j_{0}, n}^{\alpha, \alpha-1} \cdots a_{j_{i}, 2^{i}\left(n+j_{0}\right)+2^{i-1} j_{1}+\ldots+2 j_{i-1}}^{\alpha, \alpha-1} c\left(j_{0}, \ldots, j_{i}, n\right) \\
& \times \frac{2^{2 \alpha} \Gamma\left(2^{i}\left(n+j_{0}\right)+2^{i-1} j_{1}+\ldots+j_{i}+\alpha+\frac{1}{2}\right) \Gamma\left(2\left(2^{i}\left(n+j_{0}\right)+2^{i-1} j_{1}+\ldots+j_{i}\right)+1\right)}{\Gamma\left(2\left(2^{i}\left(n+j_{0}\right)+2^{i-1} j_{1}+\ldots+j_{i}\right)+\alpha+\frac{1}{2}\right) \Gamma\left(2^{i}\left(n+j_{0}\right)+2^{i-1} j_{1}+\ldots+j_{i}+1\right)} \\
& \times b_{2\left(2^{i}\left(n+j_{0}\right)+2^{i-1} j_{1}+\ldots+j_{i}\right), i+1}^{(\alpha, \alpha)} \\
& =2^{(i-1) \alpha} \sum_{j_{0}=0}^{\alpha} \ldots \sum_{j_{i}=0}^{\alpha} a_{j_{0}, n}^{\alpha, \alpha-1} \cdots a_{j_{i}, 2^{i}\left(n+j_{0}\right)+2^{i-1} j_{1}+\ldots+2 j_{i-1}}^{\alpha, \alpha-1} c\left(j_{0}, \ldots, j_{i}, n\right) \\
& \times \frac{2^{2 \alpha} \Gamma\left(2^{i}\left(n+j_{0}\right)+2^{i-1} j_{1}+\ldots+j_{i}+\alpha+\frac{1}{2}\right) \Gamma\left(2\left(2^{i}\left(n+j_{0}\right)+2^{i-1} j_{1}+\ldots+j_{i}\right)+1\right)}{\Gamma\left(2\left(2^{i}\left(n+j_{0}\right)+2^{i-1} j_{1}+\ldots+j_{i}\right)+\alpha+\frac{1}{2}\right) \Gamma\left(2^{i}\left(n+j_{0}\right)+2^{i-1} j_{1}+\ldots+j_{i}+1\right)} \\
& \times 2^{-\alpha} \sum_{j_{i+1}=0}^{\alpha} a_{j_{i+1}, 2\left(2^{i}\left(n+j_{0}\right)+2^{i-1} j_{1}+\ldots+j_{i}\right)}^{\alpha, \alpha-1} b_{2\left(2^{i}\left(n+j_{0}\right)+2^{i-1} j_{1}+\ldots+j_{i}\right)+j_{i+1}, i+1}^{(\alpha, 0)} \\
& =2^{i \alpha} \sum_{j_{0}=0}^{\alpha} \ldots \sum_{j_{i}=0}^{\alpha} \sum_{j_{i+1}=0}^{\alpha} a_{j_{0}, n}^{\alpha, \alpha-1} \cdots a_{j_{i}, 2^{i}\left(n+j_{0}\right)+2^{i-1} j_{1}+\ldots+2 j_{i-1}}^{\alpha, \alpha-1} a_{j_{i+1}, 2\left(2^{i}\left(n+j_{0}\right)+2^{i-1} j_{1}+\ldots+j_{i}\right)}^{\alpha, \alpha-1} \\
& \times c\left(j_{0}, \ldots, j_{i}, n\right) \\
& \times \frac{\left.\Gamma\left(2^{i}\left(n+j_{0}\right)+2^{i-1} j_{1}+\ldots+j_{i}+\alpha+\frac{1}{2}\right) \Gamma 2^{i+1}\left(n+j_{0}\right)+2^{i} j_{1}+\ldots+2 j_{i}+1\right)}{\Gamma\left(2^{i+1}\left(n+j_{0}\right)+2^{i} j_{1}+\ldots+2 j_{i}+\alpha+\frac{1}{2}\right) \Gamma\left(2^{i}\left(n+j_{0}\right)+2^{i-1} j_{1}+\ldots+j_{i}+1\right)} \\
& \times b_{2^{i+1}\left(n+j_{0}\right)+2^{i} j_{1}+\ldots+2 j_{i}+j_{i+1}, i+1}^{(\alpha, 0)}
\end{aligned}
$$




$$
\begin{aligned}
= & 2^{i \alpha} \sum_{j_{0}=0}^{\alpha} \ldots \sum_{j_{i+1}=0}^{\alpha} a_{j_{0}, n}^{\alpha, \alpha-1} \cdots a_{j_{i+1}, 2\left(2^{i}\left(n+j_{0}\right)+2^{i-1} j_{1}+\ldots+j_{i}\right)}^{\alpha, \alpha-1} c\left(j_{0}, \ldots, j_{i+1}, n\right) \\
& \times b_{2^{i+1}\left(n+j_{0}\right)+2^{i} j_{1}+\ldots+2 j_{i}+j_{i+1}, i+1}^{(\alpha, j)}
\end{aligned}
$$

where

$$
=\prod_{l=0}^{c\left(j_{0}, \ldots, j_{i+1}, n\right)} \frac{\Gamma\left(2^{l}\left(n+j_{0}\right)+2^{l-1} j_{1}+\ldots+j_{l}+\alpha+\frac{1}{2}\right) \Gamma\left(2^{l+1}\left(n+j_{0}\right)+2^{l} j_{1}+\ldots+2 j_{l}+1\right)}{\Gamma\left(2^{l+1}\left(n+j_{0}\right)+2^{l} j_{1}+\ldots+2 j_{l}+\alpha+\frac{1}{2}\right) \Gamma\left(2^{l}\left(n+j_{0}\right)+2^{l-1} j_{1}+\ldots+j_{l}+1\right)} .
$$

By the principle of mathematical induction, (5.2.10) holds for any $k \in \mathbb{N}$.

A common and important feature of recurrent formulas (5.2.6)-(5.2.10) is that all coefficients are nonnegative. More importantly, $k$ at the right-hand side of (5.2.10) is an arbitrary positive integer, making which large enough and combining with the following lemma help us to prove Theorems 5.1.1 and 5.1.2 in the next section.

Lemma 5.2.5. For $\alpha>-\frac{1}{2}$ and $\gamma_{0} \in \mathbb{N}_{0}$, if $\left\{\gamma_{k}, k \in \mathbb{N}\right\}$ is a sequence of bound positive rational numbers such that $2^{k} \gamma_{k} \in \mathbb{N}$ and $\lim _{k \rightarrow \infty} \gamma_{k}=\gamma>0$, then

$$
\lim _{k \rightarrow \infty} 2^{k\left(\alpha+\frac{1}{2}\right)} b_{2^{k} \gamma_{k}+\gamma_{0}, k}^{(\alpha, 0)}=2^{-\alpha-\frac{1}{2}} \int_{0}^{\pi} g(\vartheta) \vartheta^{\alpha+\frac{1}{2}} J_{\alpha-\frac{1}{2}}(\gamma \vartheta) d \vartheta .
$$

Proof. Rewriting $2^{k\left(\alpha+\frac{1}{2}\right)} b_{2^{k} \gamma_{k}+\gamma_{0}, k}^{(\alpha, 0)}$ as

$$
\begin{aligned}
& 2^{k\left(\alpha+\frac{1}{2}\right)} b_{2^{k} \gamma_{k}+\gamma_{0}, k}^{(\alpha, 0)} \\
= & \int_{0}^{\pi} g(\vartheta)\left(\frac{\vartheta}{2}\right)^{2 \alpha} 2^{-k\left(\alpha-\frac{1}{2}\right)} P_{2^{k} \gamma_{k}+\gamma_{0}}^{\left(\alpha-\frac{1}{2},-\frac{1}{2}\right)}\left(\cos \frac{\vartheta}{2^{k}}\right)\left(\frac{\sin \frac{\vartheta}{2^{k+1}}}{\frac{\vartheta}{2^{k+1}}}\right)^{2 \alpha} d \vartheta
\end{aligned}
$$

we obtain (5.2.11) from Theorem 5.1.3, by letting $k \rightarrow \infty$,

$$
\lim _{k \rightarrow \infty} 2^{k\left(\alpha+\frac{1}{2}\right)} b_{2^{k} \gamma_{k}+\gamma_{0}, k}^{(\alpha, 0)}
$$




$$
\begin{aligned}
& =\int_{0}^{\pi} g(\vartheta)\left(\frac{\vartheta}{2}\right)^{2 \alpha} \lim _{k \rightarrow \infty} 2^{-k\left(\alpha-\frac{1}{2}\right)} P_{2^{k} \gamma_{k}+\gamma_{0}}^{\left(\alpha-\frac{1}{2},-\frac{1}{2}\right)}\left(\cos \frac{\vartheta}{2^{k}}\right)\left(\lim _{k \rightarrow \infty} \frac{\sin \frac{\vartheta}{2^{k+1}}}{\frac{\vartheta}{2^{k+1}}}\right)^{2 \alpha} d \vartheta \\
& =\int_{0}^{\pi} g(\vartheta)\left(\frac{\vartheta}{2}\right)^{2 \alpha}\left(\frac{\vartheta}{2}\right)^{-\left(\alpha-\frac{1}{2}\right)} J_{\alpha-\frac{1}{2}}(\vartheta \gamma) d \vartheta \\
& =2^{-\left(\alpha+\frac{1}{2}\right)} \int_{0}^{\pi} g(\vartheta) \vartheta^{\alpha+\frac{1}{2}} J_{\alpha-\frac{1}{2}}(\vartheta \gamma) d \vartheta
\end{aligned}
$$

where the exchange between the limit and integral at the first equality is ensured by the dominated convergence theorem.

Lemma 5.2.6. For $\alpha \in \mathbb{N}_{0}, k \in \mathbb{N}_{0}$, and $n \in \mathbb{N}_{0}$,

$$
\lim _{k \rightarrow \infty} 2^{2 \alpha(k+1)} b_{n, k}^{(\alpha, 0)}=\frac{2^{\alpha-\frac{1}{2}}\left(n+\alpha-\frac{1}{2}\right) !}{n !} \lim _{x \rightarrow 0^{+}} \int_{0}^{\pi} \vartheta^{\alpha+\frac{1}{2}} \frac{J_{\alpha-\frac{1}{2}}(x \vartheta)}{x^{\alpha-\frac{1}{2}}} g(\vartheta) d \vartheta .
$$

Proof. It can be proved by the following two limiting forms, namely,

$$
\begin{aligned}
\lim _{k \rightarrow \infty} 2^{2 \alpha(k+1)} b_{n, k}^{(\alpha, 0)} & =\int_{0}^{\pi} g(\vartheta) \vartheta^{2 \alpha} \lim _{k \rightarrow \infty} P_{n}^{\left(\alpha-\frac{1}{2},-\frac{1}{2}\right)}\left(\cos \frac{\vartheta}{2^{k}}\right)\left(\frac{\sin \frac{\vartheta}{2^{k+1}}}{\frac{\vartheta}{2^{k+1}}}\right)^{2 \alpha} d \vartheta \\
& =P_{n}^{\left(\alpha-\frac{1}{2},-\frac{1}{2}\right)}(1) \int_{0}^{\pi} g(\vartheta) \vartheta^{2 \alpha} d \vartheta
\end{aligned}
$$

where $P_{n}^{\left(\alpha-\frac{1}{2},-\frac{1}{2}\right)}(1)=\left(\begin{array}{c}n+\alpha-\frac{1}{2} \\ n\end{array}\right)$ by (4.1.1) of [25], and

$$
\begin{aligned}
\lim _{x \rightarrow 0^{+}} \int_{0}^{\pi} \vartheta^{\alpha+\frac{1}{2}} \frac{J_{\alpha-\frac{1}{2}}(x \vartheta)}{x^{\alpha-\frac{1}{2}}} g(\vartheta) d \vartheta & =\int_{0}^{\pi} \vartheta^{\alpha+\frac{1}{2}} \lim _{x \rightarrow 0^{+}} \frac{J_{\alpha-\frac{1}{2}}(x \vartheta)}{x^{\alpha-\frac{1}{2}}} g(\vartheta) d \vartheta \\
& =\frac{2^{\frac{1}{2}-\alpha}}{\Gamma\left(\alpha+\frac{1}{2}\right)} \int_{0}^{\pi} g(\vartheta) \vartheta^{2 \alpha} d \vartheta
\end{aligned}
$$

which is a direct consequence of (10.7.3) of [19], $J_{\alpha}(z) \sim \frac{z^{\alpha}}{2^{\alpha} \Gamma(\alpha+1)}$ by fixing $\alpha$ and letting $z \rightarrow 0$.

\subsection{Proof of Theorems 5.1.1 and 5.1.2}

Since $g(x)$ is continuous with compact support on $[0, \infty)$ and $g(\|\mathbf{x}\|)$ is positive definite in $\mathbb{R}^{d}$, inequality (5.1.1) holds for every $x \geq 0$, i.e.,

$$
\int_{0}^{\pi} u^{\alpha+\frac{1}{2}} J_{\alpha-\frac{1}{2}}(x u) g(u) d u \geq 0, \quad x \geq 0
$$


What needs a proof is inequality (5.1.2),i.e.,

$$
b_{n}=\int_{0}^{\pi} g(\vartheta) P_{n}^{(\alpha)}(\cos \vartheta) \sin ^{2 \alpha} \vartheta d \vartheta \geq 0, \quad n \in \mathbb{N}_{0}
$$

or $b_{n}^{(\alpha, \alpha)} \geq 0$ for every $n \in \mathbb{N}_{0}$, where $\alpha=\frac{d-1}{2}$ is a nonnegative integer.

While the case $d=1$ or 3 is covered in [15], it suffices to consider $d \geq 5$ or $\alpha \in \mathbb{N}$.

To prove $b_{n}^{(\alpha, \alpha)} \geq 0$ for each $n \in \mathbb{N}$, from identity (5.2.10) we need to show

$$
b_{2^{k} n+2^{k} j_{0}+2^{k-1} j_{1}+\ldots+j_{k}, k}^{(\alpha, 0)} \geq 0, \quad j_{i}=0,1,2, \ldots, \alpha, i=1,2, \ldots, k .
$$

Since $k$ in (5.2.10) is an arbitrary positive integer, it can be chosen large enough. Noticing that

$$
2^{k} n \leq 2^{k} n+2^{k} j_{0}+2^{k-1} j_{1}+\ldots+j_{k} \leq 2^{k}(n+2 \alpha)-\alpha
$$

we can write $2^{k} n+2^{k} j_{0}+2^{k-1} j_{1}+\ldots+j_{k}$ as

$$
2^{k} n+2^{k} j_{0}+2^{k-1} j_{1}+\ldots+j_{k}=2^{k} \gamma_{k}+\gamma_{0}
$$

where $\gamma_{0} \in \mathbb{N}_{0}$ and $\gamma_{k}$ is a positive rational number bounded between $n$ and $n+2 \alpha$, so that

$$
b_{2^{k} n+2^{k} j_{0}+2^{k-1} j_{1}+\ldots+j_{k}, k}^{(\alpha, 0)}=b_{2^{k} \gamma_{k}+\gamma_{0}, k}^{(\alpha, 0)} .
$$

First, suppose that inequality (5.1.1) is strict, then the right-hand side of (5.2.11) is also positive, i.e.,

$$
\int_{0}^{\pi} g(\vartheta) \vartheta^{\alpha+\frac{1}{2}} J_{\alpha-\frac{1}{2}}(\gamma \vartheta) d \vartheta>0
$$

This is due to (5.1.1) is strict and $\gamma>0$. Then its left-hand side has to be positive. Consequently, when $\mathrm{k}$ is large enough,

$$
b_{2^{k} \gamma_{k}+\gamma_{0}, k}^{(\alpha, 0)}=b_{2^{k} n+2^{k} j_{0}+2^{k-1} j_{1}+\ldots+j_{k}, k}^{(\alpha, 0)} \geq 0,
$$

and it implies $b_{n}^{(\alpha, \alpha)} \geq 0$ by (5.2.10). Moreover, since the left-hand side of (5.2.11) is positive, there exists at least one number $k$ such that $b_{2^{k} n+2^{k} j_{0}+2^{k-1} j_{1}+\ldots+j_{k}, k}^{(\alpha, 0)}$ is positive, so that $b_{n}^{(\alpha, \alpha)}$ must be positive. 
Now we consider the case $n=0$, there are two possibilities for $2^{k} \gamma_{k}+\gamma_{0}$. If it is unbounded, then, by Lemma 5.2.5, $b_{2^{k} \gamma_{k}+\gamma_{0}, k}^{(\alpha, 0)} \geq 0$ for large $k$. If it is bounded, then it follows from Lemma 5.2.6 that $b_{2^{k} \gamma_{k}+\gamma_{0}, k}^{(\alpha, 0)} \geq 0$ when $k$ is large enough. By $(5.2 .10), b_{0}^{(\alpha, \alpha)} \geq 0$.

In a general case, (5.1.1) implies

$$
\int_{0}^{\pi} \vartheta^{\alpha+\frac{1}{2}} J_{\alpha-\frac{1}{2}}(\gamma \vartheta) g(\vartheta) d \vartheta \geq 0, \quad \gamma \geq 0
$$

For an arbitrary $\epsilon>0$, we define a function

$$
g_{\epsilon}(\vartheta)=g(\vartheta)+\epsilon(\pi-\vartheta)_{+}^{\alpha+1}, \quad \vartheta \geq 0
$$

By Theorem 7 of [11],

$$
\int_{0}^{\pi} \vartheta^{\alpha+\frac{1}{2}} J_{\alpha-\frac{1}{2}}(\gamma \vartheta)(\pi-\vartheta)_{+}^{\alpha+1} d \vartheta>0, \quad \gamma \geq 0,
$$

so that

$$
\int_{0}^{\pi} \vartheta^{\alpha+\frac{1}{2}} J_{\alpha-\frac{1}{2}}(\gamma \vartheta) g_{\epsilon}(\vartheta) d \vartheta>0, \quad \gamma \geq 0
$$

It implies

$$
\int_{0}^{\pi} g_{\epsilon}(\vartheta) P_{n}^{\left(\alpha-\frac{1}{2}, \alpha-\frac{1}{2}\right)}(\cos \vartheta)\left(\sin \frac{\vartheta}{2}\right)^{2 \alpha}\left(\cos \frac{\vartheta}{2}\right)^{2 \alpha} \geq 0, \quad n \in \mathbb{N} .
$$

Let $\epsilon \rightarrow 0$, we obtain $b_{n}^{(\alpha, \alpha)} \geq 0$ for $n \in \mathbb{N}_{0}$.

What remains is to verify that $\sum_{n=0}^{\infty} b_{n} P_{n}^{\left(\frac{d-1}{2}\right)}(1)$ converges. As is shown in [22], the continuous function $g(\vartheta)$ possesses the expansion $\sum_{n=0}^{\infty} b_{n} P_{n}^{\left(\frac{d-1}{2}\right)}(\cos \vartheta)$, which is Abel-summable for every $\vartheta \in[0, \pi]$, and, in particular, for $\vartheta=0$. Thus,

$$
\sum_{n=0}^{k} b_{n}\left|P_{n}^{\left(\frac{d-1}{2}\right)}(\cos \vartheta)\right| \leq \sum_{n=0}^{k} b_{n} P_{n}^{\left(\frac{d-1}{2}\right)}(1) \leq \lim _{r \rightarrow 1^{-}} \sum_{n=0}^{\infty} b_{n} r^{n} P_{n}^{\left(\frac{d-1}{2}\right)}(1)=g(0) .
$$

Let

$$
S_{k}=\sum_{n=0}^{k} b_{n}\left|P_{n}^{\left(\frac{d-1}{2}\right)}(\cos \vartheta)\right|
$$

Then sequence $\left\{S_{k}\right\}$ is bounded and monotone, so it is convergent. Hence the sequence

$$
S_{k}=\sum_{n=0}^{k} b_{n} P_{n}^{\left(\frac{d-1}{2}\right)}(\cos \vartheta)
$$

is absolutely and uniformly convergent for all $\vartheta \in[0, \pi]$, and converges to its Abel-sum $g(\vartheta)$. 


\section{CHAPTER 6}

\section{PLANS FOR FUTURE WORK}

In this thesis, we have reviewed the classical characterizations of isotropic positive definite functions on Euclidean spaces and spheres, and we have applied them to develop a construction of isotropic positive definite functions on spheres. However, this construction only works when the dimension of the sphere is odd. Our next work is to develop isotropic positive definite functions on all spheres. It is stated as the following conjecture.

Conjecture. Suppose that $g(x)$ is a continuous function on $[0, \infty)$, and $g(x)=0, x \geq \pi$.

For a positive integer $d$, if $g(\|\mathbf{x}\|)$ is a positive definite function in $\mathbb{R}^{d}$, then $g(\vartheta), \vartheta \in[0, \pi]$, is a positive definite function on $\mathbb{S}^{d}$. 
REFERENCES 


\section{LIST OF REFERENCES}

[1] M. Abramowitz and I. A. Stegun, Handbook of Mathematical Functions, Dover Publications, New York, 1965.

[2] R. Askey, Radial characteristic functions, Tech. Report No.1262, Math. Research Center, University of Wisconsin-Madison, 1973.

[3] H. F. Baker, Abel's theorem and the allied theory, University Press, Cambridge, 1897

[4] R. Beatson, W. zu Castell and Y. Xu, A Pólya criterion for (strict) positive-definiteness on the sphere, IMA J. Numer. Anal. 34 (2014), 550-568.

[5] S. Bochner and K. Chandrasekharan, Fourier Transforms, Princeton University Press, Princeton, 1949.

[6] D. Cheng and Y. Xiao, Excursion probability of Gaussian random fields on sphere, Bernoulli. 22 (2016), 1113-1130.

[7] K.L. Chung, A Course in Probability Theory, Academic Press, 2001

[8] F. Dai and Y. Xu, Approximation Theory and Harmonic Analysis on Spheres and Balls, Springer, New York, 2013.

[9] Stein, Elias; Weiss, Guido, Introduction to Fourier Analysis on Euclidean Spaces, N.J.: Princeton University Press, Princeton, 1971.

[10] G. E. Fasshauer, Meshfree Approximation Methods with MatLaB, World Scientific, New Jersey, 2007.

[11] G. Gasper, Positive sums of the classical orthogonal polynomials, SIAM J. Math. Anal. 8 (1977), 423-447.

[12] T. Gneiting, Strictly and non-strictly positive definite functions on spheres, Bernoulli, 2013.

[13] I. S. Gradshteyn and I. M. Ryzhik, Tables of Integrals, Series, and Products, 7th ed. Academic Press, Amsterdam, 2007.

[14] M. Jevti , D.Vukoti, M.Arsenovi, The Poisson Integral. In: Taylor Coefficients and Coefficient Multipliers of Hardy and Bergman-Type Spaces, RSME Springer Series, vol 2. Springer, Cham, 2016

[15] C. Ma, Stochastic representations of Isotropic vector random fields on spheres, Stoch. Anal. Appl. 34 (2016), 389-403. 


\section{LIST OF REFERENCES (continued)}

[16] C. Ma, Isotropic covariance matrix polynomials on spheres, Stoch. Anal. Appl. 34 (2016), 679-706.

[17] C. Ma, Time varying isotropic vector random fields on spheres, J. Theor. Probab. 30 (2017), 1763-1785.

[18] Z. Nie, C. Ma, Isotropic positvie definite functions on spheres generated from those in Euclidean spaces, Proc. Amer. Math. Soc. 147 (2019), 3047-3056.

[19] F. W. J. Olver, D. W. Lozier, R. F. Boisvert, and C. W. Clark, NIST Handbook of Mathematical Functions, Cambridge University Press, Cambridge, 2010.

[20] C. E. Rasmussen and C. K. I. Williams, Gaussian Processes for Machine Learning, Adaptive Computation and Machine Learning, The MIT Press, Massachusetts, 2006.

[21] I. J. Schoenberg, Metric spaces and completely monotone functions, Ann. Math. 39 (1938), 811-841.

[22] I. J. Schoenberg, Positive definite functions on spheres, Duke Math. J. 9 (1942), 96-108.

[23] I. N. Sneddon, The Use of Integral Transforms, New York: McGraw-Hill, 1972.

[24] P.K. Suetin, Ultraspherical polynomials, in Hazewinkel, Michiel, Encyclopedia of Mathematics, Springer Science+Business Media B.V. / Kluwer Academic Publishers, 2001.

[25] G. Szegö, Orthogonal Polynomials, AMS Coll. Publ., Vol. 23, Providence, RI, 1975.

[26] Faris, G. William, Radial functions and the Fourier transform, Department of Mathematics, University of Arizona, 2008.

[27] H. Wendland, Scattered Data Approximation, Cambridge University Press (Cambridge), 2005.

[28] Y. Xu, Positive definite functions on the unit sphere and integrals of Jacobi polynomials, Proc. Amer. Math. Soc. 146 (2018), 2039-2048. 